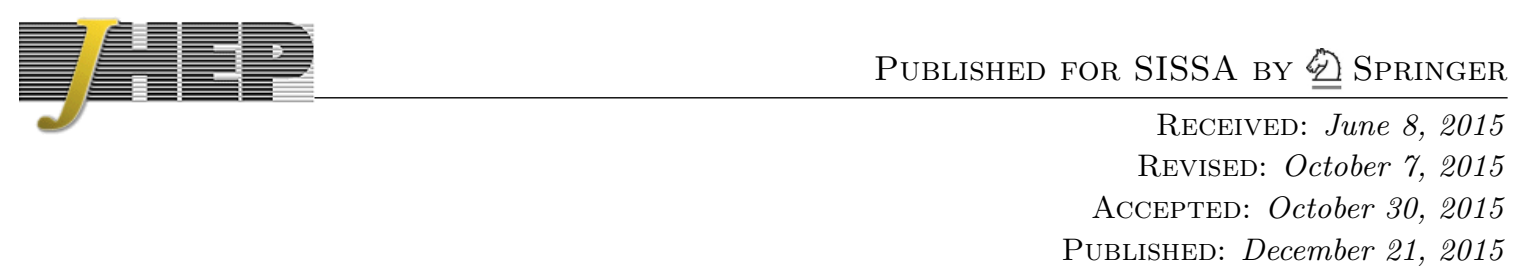

\title{
Dark Matter and gauged flavor symmetries
}

\author{
Fady Bishara, ${ }^{a, b}$ Admir Greljo, ${ }^{c, d}$ Jernej F. Kamenik, ${ }^{e, f, g}$ Emmanuel Stamou ${ }^{h}$ and \\ Jure Zupan ${ }^{a}$ \\ ${ }^{a}$ Department of Physics, University of Cincinnati, \\ Cincinnati, Ohio 45221, U.S.A. \\ ${ }^{b}$ Theoretical Physics Department, Fermilab, \\ P.O. Box 500, Batavia, IL 60510, U.S.A. \\ ${ }^{c}$ Physik-Institut, Universität Zürich, \\ CH-8057 Zürich, Switzerland \\ ${ }^{d}$ Department of Physics, University of Sarajevo, \\ Zmaja od Bosne 33-35, 71000 Sarajevo, Bosnia and Herzegovina \\ e Jožef Stefan Institute, \\ Jamova 39, 1000 Ljubljana, Slovenia \\ ${ }^{f}$ Faculty of Mathematics and Physics, University of Ljubljana, \\ Jadranska 19, 1000 Ljubljana, Slovenia \\ ${ }^{g}$ CERN TH-PH Division, \\ Meyrin, Switzerland \\ ${ }^{h}$ Department of Particle Physics and Astrophysics, Weizmann Institute of Science, \\ Rehovot 7610001, Israel \\ E-mail: bisharfy@mail.uc.edu, admir@physik.uzh.ch, \\ jernej.kamenik@cern.ch, emmanuel.stamou@weizmann.ac.il, \\ jure.zupan@cern.ch
}

ABSTRACT: We investigate the phenomenology of flavored dark matter (DM). DM stability is guaranteed by an accidental $\mathcal{Z}_{3}$ symmetry, a subgroup of the standard model (SM) flavor group that is not broken by the SM Yukawa interactions. We consider an explicit realization where the quark part of the SM flavor group is fully gauged. If the dominant interactions between DM and visible sector are through flavor gauge bosons, as we show for Dirac fermion flavored DM, then the DM mass is bounded between roughly $0.5 \mathrm{TeV}$ and $5 \mathrm{TeV}$ if the DM multiplet mass is split only radiatively. In general, however, no such relation exists. We demonstrate this using scalar flavored DM where the main interaction with the SM is through the Higgs portal. For both cases we derive constraints from flavor, cosmology, direct and indirect DM detection, and collider searches.

Keywords: Beyond Standard Model, Cosmology of Theories beyond the SM, Gauge Symmetry, Quark Masses and SM Parameters

ArXiv EPRINT: 1505.03862 


\section{Contents}

1 Introduction 1

2 Stability of flavored dark matter 2

3 Gauged flavor interactions and dark matter 4

3.1 Fermionic flavored dark matter 5

3.2 Scalar flavored dark matter 8

4 Dark matter and new physics phenomenology 9

4.1 Scan results 9

$\begin{array}{ll}4.2 & \text { Thermal relic } \\ & 11\end{array}$

$\begin{array}{lll}\text { 4.2.1 } & \text { Fermionic dark matter } & 12\end{array}$

$\begin{array}{lll}4.2 .2 & \text { Scalar dark matter } & 14\end{array}$

$\begin{array}{lll}4.3 \text { Cosmology } & 15\end{array}$

$\begin{array}{ll}\text { 4.4 Direct and indirect dark matter searches } & 17\end{array}$

$\begin{array}{ll}4.5 & \text { Searches at the LHC } \\ \end{array}$

$\begin{array}{lll}4.6 & \text { Flavor constraints } & 21\end{array}$

5 Benchmarks $\quad 22$

6 Conclusions $\quad 26$

$\begin{array}{ll}\text { A Minimal flavor violation with gauged flavor symmetries } & 29\end{array}$

B Thermal relic computation $\quad 32$

C Higgs coupling Feynman rules $\quad 35$

\section{Introduction}

The stability of dark matter (DM) is commonly assumed to be due to an exact discrete symmetry, $\mathcal{Z}_{n}$. This can either be imposed by hand or have a dynamical origin. Examples include $R$-parity in the MSSM [1], and flavor symmetries in the leptonic sector [2-6]. Here, we explore the intriguing possibility raised in refs. $[7,8]$ that the stability of DM is due to the $\mathcal{Z}_{3}$ center group of the global $\mathcal{G}_{F}^{\mathrm{SM}} \equiv \mathrm{SU}(3)_{Q} \times \mathrm{SU}(3)_{U} \times \mathrm{SU}(3)_{D}$ quark flavor symmetry. While $\mathcal{G}_{F}^{\mathrm{SM}}$ is broken by the SM Yukawa interactions, its subgroup $\mathcal{Z}_{3}$ remains unbroken in the SM. More generally, it remains exact also in the presence of New Physics (NP), if the flavor breaking is of Minimally Flavor Violating (MFV) type, i.e. only due to the SM 
Yukawas. The lightest neutral state that is odd under $\mathcal{Z}_{3}$ is therefore stable and is a DM candidate. This is the idea behind MFV dark matter [7-9].

Requiring MFV is sufficient, but not necessary. In this paper we formulate a general condition for flavored DM using flavor triality (see eq. (2.3) below). For example, any spurion in the bifundamental of $\mathcal{G}_{F}^{\mathrm{SM}}$ leaves the above $\mathcal{Z}_{3}$ unbroken. The flavor breaking can thus be quite far from MFV and still have stability of DM guaranteed by the flavor dynamics. To illustrate this point we consider the model of ref. [10] where the flavorbreaking spurions have the form $Y_{u, d}^{-1}$ and are thus canonically not of the MFV type. In this model the SM quark flavor symmetry $\mathcal{G}_{F}^{\mathrm{SM}}$ is fully gauged giving rise to flavor-gauge bosons, the Yukawas are promoted to physical scalar fields (flavons) transforming under flavor, and in addition there is a set of chiral fermions that cancel the anomalies in the flavor-gauge sector.

Using this renormalizable model we show below that a thermal relic DM can be in a nontrivial representation of $\mathcal{G}_{F}^{\mathrm{SM}}$. There are two conflicting constraints on this setup. On the one hand, Flavor Changing Neutral Current (FCNC) constraints impose lower bounds on the masses of states in nontrivial flavor representations. On the other hand, a DM relic density consistent with observations requires large enough DM annihilation cross section so that some of these same particles need to be sufficiently light. Both of these requirements are satisfied for $\mathcal{O}(\mathrm{TeV}) \mathrm{DM}$ mass. This is low enough that it may be tested by direct and indirect DM detection experiments and searched for at high-energy particle colliders.

While the phenomenology of flavored DM models can be found in refs. [8, 11-21], the construction of an explicit renormalizable model with inclusion of flavor-gauge interactions is new. Within our framework, the constraints on DM are more severe compared to a generic Effective Field Theory (EFT) analysis [7, 9]. In particular, the flavor constraints from new fermionic states, and the fact that the vacuum expectation values (vevs) of the flavon fields need to reproduce the quark masses, makes the structure of the theory much more rigid and predictive.

The paper is structured as follows. In section 2 we derive general conditions for DM to be stabilized by the exact accidental flavor symmetry of the SM (flavor triality). An explicit realization of this possibility is introduced in section 3 in the form of a model with fully gauged $\mathcal{G}_{F}^{\mathrm{SM}}$. The resulting DM, flavor, and collider phenomenology is analyzed in detail in section 4 . We summarize our conclusions in section 6 , while more technical details of some of our computations are relegated to the appendices.

\section{Stability of flavored dark matter}

We start by formulating the general conditions required for flavored DM to be stable due to flavor triality. The SM exhibits a large global flavor symmetry $\mathrm{U}(3)_{Q} \times \mathrm{U}(3)_{U} \times \mathrm{U}(3)_{D} \times$ $\mathrm{U}(3)_{L} \times \mathrm{U}(3)_{E}$ in the limit of vanishing Yukawa interactions. In this paper we focus on the quark sector. This has the global symmetry $\mathcal{G}_{F}^{\mathrm{SM}} \times \mathrm{U}(1)_{Y} \times \mathrm{U}(1)_{B} \times \mathrm{U}(1)_{\mathrm{PQ}}$. The three $\mathrm{U}(1)$ factors are the hypercharge, baryon number $(B)$, and the Peccei-Quinn symmetry, respectively. The remaining semisimple group is $\mathcal{G}_{F}^{\mathrm{SM}}=\mathrm{SU}(3)_{Q} \times \mathrm{SU}(3)_{U} \times \mathrm{SU}(3)_{D}$. The 
SM quarks transform under it as

$$
Q_{L} \sim(3,1,1), \quad U_{R} \sim(1,3,1), \quad D_{R} \sim(1,1,3) .
$$

The $\mathcal{G}_{F}^{\mathrm{SM}}$ global symmetry is broken by the SM Yukawa terms

$$
\mathcal{L}_{Y}=\bar{Q}_{L} \tilde{H} y_{u} U_{R}+\bar{Q}_{L} H y_{d} D_{R}+\text { h.c. },
$$

where $\tilde{H}=i \sigma_{2} H^{*} . \mathcal{L}_{Y}$ is formally invariant under $\mathcal{G}_{F}$ if $y_{u, d}$ are promoted to spurions that transform as $(3, \overline{3}, 1)$ and $(3,1, \overline{3})[22-28]$. NP is of MFV type if $y_{u, d}$ are the only flavor-breaking spurions also in the NP sector.

The SM Yukawa couplings in eq. (2.2) break $\mathrm{U}(1)_{\mathrm{PQ}}$ and break $\mathcal{G}_{F}^{\mathrm{SM}}$ to its center group $\mathcal{Z}_{3}^{U D Q}$, under which all three generations of quarks transform as

$\left\{U_{R}, D_{R}, Q_{L}\right\} \rightarrow e^{i 2 \pi / 3}\left\{U_{R}, D_{R}, Q_{L}\right\}$. In the SM the $\mathcal{Z}_{3}^{U D Q}$ is identical to a subgroup of $\mathrm{U}(1)_{B}$. This is no longer true in the presence of NP. In MFV for instance, $\mathcal{Z}_{3}^{U D Q}$ remains exact, while $\mathrm{U}(1)_{B}$ can be broken, e.g., by dimension-9 operators [29] (see also [8]).

The $\mathcal{Z}_{3}^{U D Q}$ may be the underlying reason for the stability of DM. To make this explicit it is useful to introduce $\mathcal{Z}_{3}^{\chi}$, a diagonal subgroup of $\mathcal{Z}_{3}^{U D Q} \times \mathcal{Z}_{3}^{c}$. Here, $\mathcal{Z}_{3}^{c}$ is the center group of color $\mathrm{SU}(3)_{c}$, under which $\left\{U_{R}, D_{R}, Q_{L}\right\} \rightarrow e^{-i 2 \pi / 3}\left\{U_{R}, D_{R}, Q_{L}\right\}$. All the SM fields are thus $\mathcal{Z}_{3}^{\chi}$ singlets. In MFV NP $\mathcal{Z}_{3}^{\chi}$ is exact, so that the lightest $\mathcal{Z}_{3}^{\chi}$ odd particle is stable and can be a DM candidate [8].

We generalize this observation beyond MFV. To this end, we introduce the notion of flavor triality [8]. Consider a field $X$ in the $\mathcal{G}_{F}^{\mathrm{SM}}$ representation $X \sim\left(n_{Q}^{X}, m_{Q}^{X}\right) \times\left(n_{U}^{X}, m_{U}^{X}\right) \times$ $\left(n_{D}^{X}, m_{D}^{X}\right)$, where $n_{i}^{X}, m_{i}^{X}$ are the Dynkin coefficients of the corresponding $\mathrm{SU}(3)_{i}$ group. We call flavor triality the quantity

$$
\left(n_{X}-m_{X}\right) \bmod 3
$$

where $n_{X}=n_{Q}^{X}+n_{U}^{X}+n_{D}^{X}$ and $m_{X}=m_{Q}^{X}+m_{U}^{X}+m_{D}^{X}$.

The basic requirements for flavored DM to be stable due to $\mathcal{Z}_{3}^{\chi}$ are the following. First of all, $\mathcal{G}_{F}^{\mathrm{SM}}$ needs to be a good symmetry in the UV. Secondly, $\mathcal{G}_{F}^{\mathrm{SM}}$ needs to be broken only by spurions $\langle\Phi\rangle$ with zero flavor triality, $\left(n_{\langle\Phi\rangle}-m_{\langle\Phi\rangle}\right) \bmod 3=0$. This ensures that $\mathcal{Z}_{3}^{\chi}$ is unbroken. (The spurions $\langle\Phi\rangle$ need to be color singlets in order not to break color.) The lightest $\mathcal{Z}_{3}^{\chi}$ odd state is then stable. If it is a color singlet it is a potential DM candidate. This also means that DM is in a nontrivial flavor representation with nonzero flavor triality, $\left(n_{\chi}-m_{\chi}\right) \bmod 3 \neq 0$.

The above shows that models with flavored DM can deviate significantly from MFV. In particular, $\mathcal{Z}_{3}^{\chi}$ is not broken by a vev of any field that is in an adjoint or in a bifundamental of $\mathcal{G}_{F}^{\mathrm{SM}}$. Specifically, any function $f\left(y_{u}, y_{d}\right)$ leaves $\mathcal{Z}_{3}^{\chi}$ unbroken. More generally, additional flavor-breaking sources that transform as $(8,1,1),(1,3, \overline{3}), \ldots$ may be present without spoiling DM stability. While the flavor structure of such NP models is not of MFV type in general, the stability of DM is still a consequence of an unbroken flavor subgroup. DM is in a nontrivial representation of the flavor group, leading to distinct phenomenology depending on the nature of the flavor breaking and on which flavor multiplet $\chi$ belongs to. 
An important starting point in the above discussion was the assumption that $\mathcal{G}_{F}^{\mathrm{SM}}$ is a good symmetry in the UV. This is most easily achieved, if $\mathcal{G}_{F}^{\mathrm{SM}}$ is gauged. We explore this possibility in the remainder of the paper.

\section{Gauged flavor interactions and dark matter}

We gauge the full SM quark-flavor symmetry $\mathcal{G}_{F}^{\text {SM }}$. The fermionic sector is extended to cancel the anomalies of the new gauge sector. We use the model of ref. [10] that allows for $\mathcal{O}(\mathrm{TeV})$ flavor gauge bosons (FGBs). The SM Yukawas, $y_{u, d}$, arise from the vevs of new scalar fields transforming as

$$
Y_{u} \sim(\overline{3}, 3,1), \quad Y_{d} \sim(\overline{3}, 1,3),
$$

under $\mathcal{G}_{F}^{\mathrm{SM}}$. The minimal set of chiral fermions that ensures anomaly cancellation of the new gauged sector is

$$
\Psi_{u R} \sim(3,1,1), \quad \Psi_{d R} \sim(3,1,1), \quad \Psi_{u L} \sim(1,3,1), \quad \Psi_{d L} \sim(1,1,3),
$$

where the index $L$ and $R$ represents their chirality. Together with the SM fermions they, therefore, form vector-like representations of $\mathcal{G}_{F}^{\mathrm{SM}}$. The SM gauge quantum numbers of $\Psi_{u R}, \Psi_{d R}, \Psi_{u L}, \Psi_{d L}$ are the same as for $U_{R}, D_{R}, U_{R}, D_{R}$, respectively, i.e., they are $\mathrm{SU}(2)_{L}$ singlets but charged under $\mathrm{U}(1)_{Y}$. Because the new fermions are vector-like under the SM, e.g, $\Psi_{u R}$ transforms like $\Psi_{u L}$ under the SM, all anomalies in the SM sector cancel. Remarkably, with the above fermionic content also all mixed gauge anomalies cancel. In fact, one could also gauge two additional flavor diagonal $\mathrm{U}(1)$ 's, $\mathrm{U}(1)_{U}$ and $\mathrm{U}(1)_{D}$, as well as $\mathrm{U}(1)_{B-L}$, a possibility that we do not pursue further, but is discussed in ref. [10].

The Yukawa and relevant mass terms in the Lagrangian are [10]

$$
\begin{aligned}
\mathcal{L}_{\text {mass }} \supset \lambda_{u} \bar{Q}_{L} \tilde{H} \Psi_{u R}+\lambda_{u}^{\prime} \bar{\Psi}_{u L} Y_{u} \Psi_{u R}+M_{u} \bar{\Psi}_{u L} U_{R} \\
+\lambda_{d} \bar{Q}_{L} H \Psi_{d R}+\lambda_{d}^{\prime} \bar{\Psi}_{d L} Y_{d} \Psi_{d R}+M_{d} \bar{\Psi}_{d L} D_{R}+\text { h.c. }
\end{aligned}
$$

where $\lambda_{u, d}^{(')}$ are flavor-universal coupling constants and $M_{u, d}$ flavor-universal mass parameters. The mass terms in eq. (3.3) mix the states $\Psi_{u L, u R}$ and $U_{L, R}$ forming mass eigenstates $u_{i}$ and $u_{i}^{\prime}$, where $i=1,2,3$ is the generation index (and similarly for down-quark states). After electroweak symmetry breaking the masses for the two mass-eigenstate sets are, in the limit $m_{u_{i}^{\prime}} \gg m_{u_{i}},[10,30]$

$$
m_{u^{i}} \approx \frac{v}{\sqrt{2}} \frac{\lambda_{u} M_{u}}{\lambda_{u}^{\prime}\left\langle Y_{u}\right\rangle_{i}}, \quad m_{u_{i}^{\prime}} \approx \lambda_{u}^{\prime}\left\langle Y_{u}\right\rangle_{i} .
$$

The mass matrix for the FGBs, $A_{A}^{a}, A=Q, U, D$ and $a=1, \ldots, 8$, is governed by the vevs of the $Y_{u, d}$ scalar fields and the gauge couplings, $[10,30]$

$$
\left(\mathcal{M}_{A B}^{2}\right)_{a b}=\frac{1}{4} g_{A} g_{B} \operatorname{Tr}\left[\left\langle Y_{u}\right\rangle\left\{\lambda^{a}, \lambda^{b}\right\}\left\langle Y_{u}\right\rangle^{\dagger}\right]\left(\delta_{A B} \delta_{A Q}-2 \delta_{A Q} \delta_{B U}+Q \leftrightarrow U\right)+U, u \leftrightarrow D, d,
$$


with $\lambda^{a}$ the Gell-Mann SU(3) matrices. The mass matrix is $24 \times 24$ dimensional. We denote the mass-ordered eigenstates by $A^{m}, m=1, \ldots, 24$, where $A^{24}$ is the lightest one. The lightest gauge boson is found to be along the three diagonal $\lambda_{8}$ directions. This is a consequence of $\left\langle Y_{u}\right\rangle$ and $\left\langle Y_{d}\right\rangle$ being almost aligned and with very hierarchical eigenvalues, where the $\left\langle Y_{u}\right\rangle_{33}$ and $\left\langle Y_{d}\right\rangle_{33}$ are the smallest eigenvalues.

The SM Yukawas, $y_{u, d}$, are generated after $Y_{u, d}$ obtain a vev and the $\Psi_{i}$ fields are integrated out. To first order in $M_{u, d} /\left\langle Y_{u, d}\right\rangle$, this gives

$$
y_{u} \simeq \frac{\lambda_{u} M_{u}}{\lambda_{u}^{\prime}\left\langle Y_{u}\right\rangle}, \quad y_{d} \simeq \frac{\lambda_{d} M_{d}}{\lambda_{d}^{\prime}\left\langle Y_{d}\right\rangle}
$$

The SM Yukawas, $y_{u, d}$, are non-analytic functions of the spurions $\left\langle Y_{u, d}\right\rangle$, which signals that the theory is strictly speaking not MFV. Analogously, the NP states, $u_{i}^{\prime}, d_{i}^{\prime}$ and $A^{m}$, have masses that are non-analytic in terms of the SM Yukawas. However, the low-energy observables, with only the SM fields on the external legs can be MFV-like. If the $M_{u, d} /\left\langle Y_{u, d}\right\rangle$ suppressed terms are kept in eq. (3.6), the $y_{u, d}$ become more involved functions of $\left\langle Y_{u, d}\right\rangle^{-1}$. These are analytic in $\left\langle Y_{u, d}\right\rangle^{-1}$ since the effects of NP states decouple in the $\left\langle Y_{u, d}\right\rangle \rightarrow \infty$ limit. Similarly, the NP contributions to the low-energy observables $C_{i}$ take the form $\delta C_{i}=F\left(\left\langle Y_{u}\right\rangle^{-1},\left\langle Y_{d}\right\rangle^{-1}\right)=\tilde{F}\left(y_{u}, y_{d}\right)$, with $F, \tilde{F}$ analytic functions. One can thus expand

$\delta C_{i}=a_{1} y_{u} y_{u}^{\dagger}+a_{2}\left(y_{u} y_{u}^{\dagger}\right)^{2}+b_{1} y_{u} y_{u}^{\dagger} y_{d} y_{d}^{\dagger}+\cdots$, where we assumed for illustration that the transition is due to the left-handed quark current. As long as there are no large flavorconserving ratios, i.e., as long as $\left(\lambda_{u} M_{u} / \lambda_{u}^{\prime}\right) /\left(\lambda_{d} M_{d} / \lambda_{d}^{\prime}\right) \ll 1 /\left|V_{c b}\right|$, the Taylor expansion can be truncated after a few terms (see ref. [28] for a more detailed discussion). In this limit, the low-energy effects are of the MFV type, suppressing the FCNCs to acceptable levels already for NP states at the electroweak scale. In a numerical analysis that we perform in appendix A, we find that the expansion of the effective weak Hamiltonian in terms of SM Yukawa couplings can indeed still be performed for FGB contributions.

Since $\left\langle Y_{u, d}\right\rangle$ are in the bifundamental representation of $\mathcal{G}_{F}^{\mathrm{SM}}$, the $\mathcal{Z}_{3}^{\chi}$ remains unbroken. As argued above the $\mathcal{Z}_{3}^{\chi}$ can be used to make flavored DM candidates stable. We consider two examples: i) a fermionic $\mathrm{DM}$ in a vector-like representation of $\mathcal{G}_{F}^{\mathrm{SM}}$ that thermalizes with the visible sector through FGBs, and ii) a scalar flavored DM, that interacts with the visible sector by exchanging FGBs as well as the Higgs.

It is important to note that generating the hierarchy in the SM Yukawa couplings (eq. (3.6)) requires a large hierarchy in the vevs of the flavons [10]. And, while the fermion masses in the SM are technically natural, the new scalar sector may not be.

\subsection{Fermionic flavored dark matter}

The DM in the first model is a massive Dirac fermion, $\chi$, in a vector-like representation of $\mathrm{SU}(3)_{U}$

$$
\chi \sim(1,3,1),
$$

so that no gauge anomalies are induced. Its mass term is

$$
\mathcal{L}_{\text {mass }}^{\chi}=m_{\chi}^{0} \bar{\chi} \chi .
$$




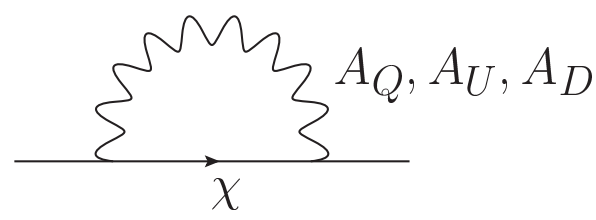

Figure 1. Radiative corrections due to FGBs, $A_{Q}, A_{u}, A_{D}$, split the DM multiplet $\chi$.

Since $\chi$ is charged under $\mathcal{Z}_{3}^{\chi}$, the lightest member of the $\chi$ triplet is stable. Note that we could also gauge a larger global group $\mathcal{G}_{F}^{S M} \times \mathrm{SU}(3)_{\chi}$, with $\chi$ transforming under $\mathrm{SU}(3)_{\chi}$. That we identify $\mathrm{SU}(3)_{\chi}$ with $\mathrm{SU}(3)_{U}$ is a model-building choice.

The DM flavor triplet, $\chi$, is split by radiative corrections due to the exchanges of FGBs, see figure 1. In the $m_{\chi}^{0} \ll m_{A}$ limit, the DM mass splitting at one-loop is given by

$$
\Delta m_{\chi}=-\frac{3}{4} \frac{g_{U}^{2}}{16 \pi^{2}} m_{\chi}^{0}\left(\Xi-\frac{1}{3} \operatorname{Tr} \Xi\right)
$$

where $\Delta m_{\chi}$ is a $3 \times 3$ matrix and so is $\Xi \equiv \lambda^{a}\left(\log \mathcal{M}_{A}^{2} / \mu^{2}\right)^{a b} \lambda^{b}$. The FGB mass matrix $\mathcal{M}_{A}^{2}$ is given in eq. (3.5), while the $a, b$ indices run only over the eight $\mathrm{SU}(3)_{U}$ generators. The unphysical $\mu$ dependence cancels in the r.h.s. of eq. (3.9). The $\chi_{i}, i=1,2,3$, mass eigenstates are obtained by diagonalizing the mass matrix $\Delta m_{\chi}$. And $\Xi$ is a function of $Y_{u}^{\dagger} Y_{u}$ and $Y_{u}^{\dagger} Y_{d} Y_{d}^{\dagger} Y_{u}$ vev combinations, making the $\chi$ mass eigen-basis slightly misaligned with respect to the up-quark one. The $\chi_{1}$ mass receives contributions from the heaviest FGBs (cf. section 5). The lightest state is thus $\chi_{1}$, i.e., with the predominantly up-quark flavor, while the heaviest is the top-flavored state, $\chi_{3}$.

In the numerics we use the exact one-loop expressions for the DM mass splitting,

$$
\Xi=\frac{3}{2} \lambda^{a} \mathcal{W}^{a+8, m}\left[B_{1}\left(m_{\chi}^{2}, m_{A_{m}}^{2}, m_{\chi}^{2}\right)-B_{0}\left(m_{\chi}^{2}, m_{A_{m}}^{2}, m_{\chi}^{2}\right)\right] \mathcal{W}^{\dagger^{m, c+8}} \lambda^{c}
$$

Summation over FGB mass eigenstate indices $m=1, \ldots, 24$ and over $a, c=1, \ldots, 8$ is understood. The $24 \times 24$ dimensional matrix $\mathcal{W}$ diagonalizes the gauge-boson mass matrix and $B_{0,1}$ are the Veltman-Passarino functions. Typical values of the mass splitting as a function of $g_{U}$ are shown in figure 2. Denoting $\Delta m_{i j} \equiv m_{\chi_{i}}-m_{\chi_{j}}$, we see that $\Delta m_{32} \ll \Delta m_{31}$, so that the lightest state $\chi_{1}$ is split away from $\chi_{2}$ and $\chi_{3}$, with the latter approximately degenerate, $m_{\chi_{2}} \simeq m_{\chi_{3}}$. This is very different from MFV DM [7-9] where the DM mass splitting is assumed to be expandable in the SM Yukawa couplings. In that case one has an approximate $\mathrm{U}(2)$ symmetry for the first two generations giving $m_{\chi_{1}} \simeq m_{\chi_{2}}$, while the top-flavored DM, $\chi_{3}$, is split away from the first two generations, and can be either significantly heavier or lighter.

The relation $\Delta m_{32} \ll \Delta m_{31}$ signifies that our flavored DM is non-MFV. The flavor gauge group $\mathrm{SU}(3)_{U}$ is broken by the FGB vevs $\left\langle Y_{u}\right\rangle$. This breaking is larger in the first two generations. Since the quark masses are inversely proportional to $\left\langle Y_{u}\right\rangle$, this leads to an approximate global $\mathrm{U}(2)_{U}$ symmetry in the quark sector. Such an approximate symmetry is not found for the radiative corrections to DM masses, $m_{\chi_{i}}$. The DM multiplet has a chiral symmetry in the limit $m_{\chi} \rightarrow 0$ ensuring that the radiative corrections are proportional to 


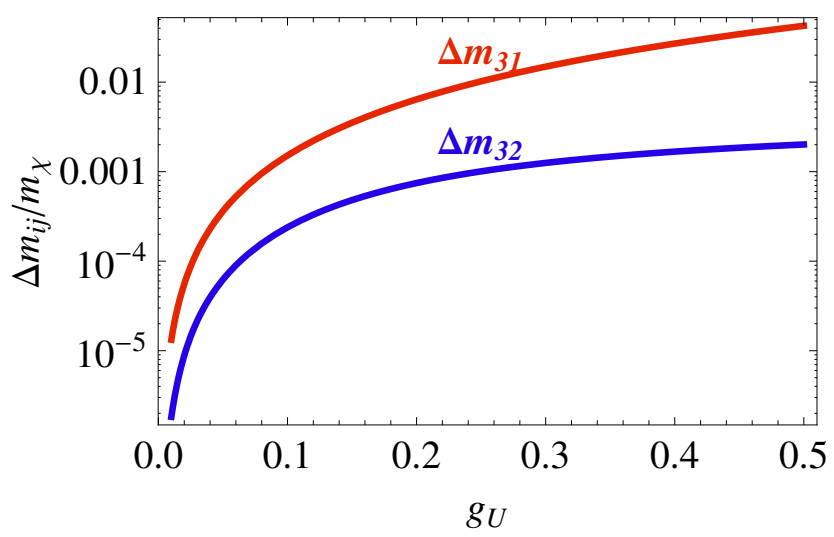

Figure 2. Typical radiative splitting of the fermionic DM multiplet with $\Delta m_{31}\left(\Delta m_{32}\right)$ shown in red (blue) as a function of $g_{U}$, while all other parameters are kept fixed at $g_{Q}=0.4, g_{D}=0.5$, $M_{u}=600 \mathrm{GeV}, M_{d}=400 \mathrm{GeV}, \lambda_{u}=1, \lambda_{u}^{\prime}=0.5, \lambda_{d}=0.25, \lambda_{d}^{\prime}=0.3$.

$m_{\chi}$ and only log-dependent on FGB masses. The splitting does not vanish in the $\left\langle Y_{u}\right\rangle \rightarrow \infty$ limit (or, equivalently, $y_{u} \rightarrow 0$ limit), since in this limit the $\mathrm{SU}(3)_{U}$ gauge group is still completely broken. Numerically, for $m_{\chi} \sim \mathrm{TeV}$ the splittings are $\Delta m_{31} \sim \mathcal{O}(10 \mathrm{GeV})$ and $\Delta m_{32} \sim \mathcal{O}(1 \mathrm{GeV})$ for $g_{U} \sim 0.4$ and can be less than a pion mass for an order of magnitude smaller $g_{U}$.

The DM multiplet can be split more significantly if there is flavor breaking beyond $\left\langle Y_{u}\right\rangle,\left\langle Y_{d}\right\rangle$. As an example we consider an additional scalar field in the adjoint of $\mathrm{SU}(3)_{U}$, $\Phi_{U} \sim(1,8,1)$. The DM mass Lagrangian now reads

$$
\mathcal{L}_{\text {mass }}^{\prime}=m_{\chi}^{0} \bar{\chi}_{L} \chi_{R}+\lambda_{\chi} \bar{\chi}_{L} \Phi_{U} \chi_{R}+\text { h.c. },
$$

and yields DM masses that are split already at tree level, $\Delta m_{\chi}=\lambda_{\chi}\left\langle\Phi_{U}\right\rangle$. We assume that $\left\langle\Phi_{U}\right\rangle$ is aligned with $Y_{U}^{\dagger} Y_{U}$. Then the two diagonalize in the same basis giving $\mathcal{O}(1)$ splitting between all three members of the multiplet. The alignment is not needed in general, but does simplify our analysis. For the same reason, we also take the first state to be the lightest one, $m_{\chi_{1}}<m_{\chi_{2,3}}$.

The $\chi_{i}$ interact with the SM through FGBs. This also induces the decay of the heavier two states in the DM multiplet, $\chi_{2,3}$, to $\chi_{1}$. We parametrize the relevant interactions with

$$
\begin{aligned}
\mathcal{L}_{\chi} \supset & \left(\hat{g}_{\chi}^{m}\right)_{i j} \bar{\chi}_{i} \gamma^{\mu} \chi_{j} A_{\mu}^{m} \\
& +\bar{u}_{k} \gamma^{\mu}\left(\left(\hat{\mathcal{G}}_{R}^{u}\right)_{k l, m} P_{R}+\left(\hat{\mathcal{G}}_{L}^{u}\right)_{k l, m} P_{L}\right) u_{l} A_{\mu}^{m} \\
& +\bar{d}_{k} \gamma^{\mu}\left(\left(\hat{\mathcal{G}}_{R}^{d}\right)_{k l, m} P_{R}+\left(\hat{\mathcal{G}}_{L}^{d}\right)_{k l, m} P_{L}\right) d_{l} A_{\mu}^{m},
\end{aligned}
$$

where $P_{R, L} \equiv \frac{1}{2}\left(1 \pm \gamma^{5}\right)$ and $k, l=1, \ldots, 6$. The couplings of $\chi_{i}$ to the gauge bosons are

$$
\left(\hat{g}_{\chi}^{m}\right)_{j i}=\left(-\frac{1}{2} g_{U} U^{\dagger} \lambda_{n}^{U} \mathcal{W}^{n m} U\right)_{j i}
$$

where $U$ diagonalizes the $m_{\chi}$ mass matrix, $U^{\dagger} m_{\chi} U=\hat{m}_{\chi}$, and $\mathcal{W}$ diagonalizes the gauge-boson mass matrix. The explicit form of FGB couplings to exotic and SM quarks, $\left(\hat{\mathcal{G}}_{L / R}^{u / d}\right)_{k l, m}$, can be found in appendix A.2 of ref. [30]. 
The partial decay width for $\chi_{i} \rightarrow \chi_{j} q_{l} \bar{q}_{k}$ is, neglecting hadronization effects,

$$
\Gamma\left(\chi_{i} \rightarrow \chi_{j} q_{l} \bar{q}_{k}\right)=\frac{3}{(2 \pi)^{3}} \frac{\Delta m_{i j}^{5}}{15}\left[\left|\sum_{m}\left(\hat{g}_{\chi}^{m}\right)_{i j} \frac{1}{m_{A^{m}}^{2}}\left(\hat{\mathcal{G}}_{L}^{u}\right)_{k l, m}\right|^{2}+L \rightarrow R\right],
$$

where the sum runs over the FGB mass eigenstates $m=1, \ldots, 24$. Expression (3.14) is valid in the $\left|\Delta m_{i j}\right| \ll m_{\chi_{i}}$ limit, and neglecting the quark masses. The above approximations are valid for all the values of parameter for which the correct relic abundance is obtained and the FCNC, collider and direct DM detection constraints are satisfied.

If the mass splitting is less than the pion mass, the decay $\chi_{i} \rightarrow \chi_{j} q_{l} \bar{q}_{k}$ is kinematically not allowed. The heavier $\chi_{i}$ states then decay radiatively to one or two photons. The one-photon decay, $\chi_{i} \rightarrow \chi_{j} \gamma$, first arises at the two-loop level and the two photon decay, $\chi_{i} \rightarrow \chi_{j} \gamma \gamma$, at one-loop. For our purposes order of magnitude estimates of the decay widths suffice. The naive dimensional analysis estimate for the one-loop two-photon decay gives,

$\Gamma\left(\chi_{i} \rightarrow \chi_{j} \gamma \gamma\right) \sim \frac{\Delta m_{i j}^{9}}{8 \pi} \frac{1}{16 \pi^{2}}\left(\frac{\alpha}{4 \pi}\right)^{2}\left[\left|\sum_{m, f} \frac{\left(\hat{g}_{\chi}^{m}\right)_{i j}}{m_{A^{m}}^{2}} \frac{Q_{u}^{2}}{m_{f}^{2}}\left(\left(\hat{\mathcal{G}}_{L}^{u}\right)_{f f, m}-\left(\hat{\mathcal{G}}_{R}^{u}\right)_{f f, m}\right)\right|^{2}+u \rightarrow d\right]$

where $Q_{u}=2 / 3$ and $Q_{d}=-1 / 3$ are the electromagnetic charges of up and down quarks. The sum over $m$ runs over the FGB mass eigenstates, while the sum over $f$ is over the SM quarks and exotic states, of mass $m_{f}$ (for up, down and strange quarks this needs to be replaced with $\left.\Lambda_{\mathrm{QCD}}\right)$. While, for the two-loop one-photon decay we obtain,

$\Gamma\left(\chi_{i} \rightarrow \chi_{j} \gamma\right) \sim \frac{\Delta m_{i j}^{3}}{8 \pi} \frac{\alpha}{(4 \pi)^{7}}\left[\left|\sum_{m, f}\left(\hat{g}_{\chi}^{m}\right)_{i i}\left(\hat{g}_{\chi}^{m}\right)_{i j} \frac{m_{i} Q_{u}}{m_{A^{m}}^{2}}\left(\left(\hat{\mathcal{G}}_{L}^{u}\right)_{f f, m}^{2}-\left(\hat{\mathcal{G}}_{R}^{u}\right)_{f f, m}^{2}\right)\right|^{2}+u \rightarrow d\right]$.

\subsection{Scalar flavored dark matter}

The second model has scalar DM, $\phi$, in a fundamental representation of $\mathrm{SU}(3)_{U}$

$$
\phi \sim(1,3,1)
$$

The main difference with the fermionic flavored DM from the previous subsection is that the scalar DM interacts with the visible sector via two different types of interactions. The first is its couplings to the FGBs, which is similar to the case of the fermionic DM. The second is a direct coupling to the Higgs

$$
\mathcal{L}_{\text {int }}^{\mathrm{DM}}=\lambda_{H}\left(\phi^{\dagger} \phi\right)\left(H^{\dagger} H\right) .
$$

For a thermal relic the DM annihilations proceed predominantly through the Higgs portal. The interactions via FGBs are subdominant except if $m_{\phi} \simeq m_{A}^{a} / 2$ for some $A^{a}$. Unless this is the case, the fact that the DM carries a flavor quantum number is exhibited only through the multiplicity of the states. 
After electroweak symmetry breaking, the DM-Higgs interactions are given by

$$
\mathcal{L}_{\text {int }}^{\mathrm{DM}} \supset \lambda_{H}\left(v h+v^{2} / 2\right) \phi^{\dagger} \phi,
$$

and the DM mass term $m_{0}^{2} \phi^{\dagger} \phi$ is shifted by the Higgs condensate to give the DM mass of

$$
m_{\phi}^{2}=m_{0}^{2}+v^{2} / 2
$$

The vevs of the flavons, $\left\langle Y_{u}\right\rangle$ and $\left\langle Y_{d}\right\rangle$, split the DM multiplet at tree level through

$$
\mathcal{L} \supset \kappa_{1}\left(\phi^{\dagger} \lambda^{a} \phi\right) \operatorname{Tr}\left(Y_{u}^{\dagger} \lambda^{a} Y_{u}\right)+\kappa_{2}\left(\phi^{\dagger}\left\{\lambda^{a}, \lambda^{b}\right\} \phi\right) \operatorname{Tr}\left(Y_{u}^{\dagger}\left\{\lambda^{a}, \lambda^{b}\right\} Y_{u}\right) .
$$

The spectrum is also split by radiative corrections due to FGBs. These are quadratically divergent and proportional to the square of the FGB mass. In principle, it is possible to fine tune the tree-level and loop contributions to give almost degenerate DM flavor multiplet. However, given the hierarchical FGB masses, it is more likely that the DM multiplet is split completely, and only the lightest state is relevant for DM phenomenology. Depending on the signs of $\kappa_{i}$ in eq. (3.21) the lightest $\phi$ component can be either top-quark or upquark flavored. We choose the latter option in the numerics for easier comparison with the fermionic case.

\section{Dark matter and new physics phenomenology}

We turn next to the phenomenology of the flavored DM models. We perform a scan over the parameters of the models and show that the lowest DM states, both for the fermionic DM, $\chi$, and the scalar DM, $\phi$, can be thermal relics. To make the scan numerically tractable we rely on several approximations in calculating the relic density, which we explain below. We also discuss the predictions for direct DM detection, and the constraints from FCNCs and collider searches.

\subsection{Scan results}

In the scan we fix $\lambda_{u}=1$ and vary $\lambda_{d} \in[1 /(4 \pi), 1]$. The range is chosen with the expectation that one will be able to accommodate both the SM top and bottom quark Yukawas as well as satisfy electroweak precision constraints and direct $t^{\prime}$ and $b^{\prime}$ searches [10]. In addition we vary conservatively $\lambda_{u, d}^{\prime} \in\left[1 /(4 \pi)^{2}, 4 \pi\right], g_{Q, U, D} \in\left[1 /(4 \pi)^{2}, 4 \pi\right]$, and $M_{u, d} \in$ $[0.2,20] \mathrm{TeV}$. To a good approximation, the variation of $M_{u}$ effectively compensates the fact that we do not vary $\lambda_{u}$ as seen from eq. (3.6). We have verified that further extending these parameter ranges does not extend the viable DM-model parameter space. For instance, the upper ranges of $g_{Q, U, D}$ and $\lambda_{u, d}^{\prime}$ already lie in the non-perturbative regime. To ensure perturbative control we require that all the FGB decay widths satisfy $\Gamma_{A^{m}}<0.5 m_{A^{m}}$, and that the radiative mass splitting for the fermionic DM is $\left|\Delta m_{i j}\right|<0.5 m_{\chi}$. This imposes upper bounds on $g_{Q, U, D}$ that are typically close to $\sqrt{4 \pi}$. Similar constraints on $\lambda_{u, d}^{\prime}$ are expected to follow from analogous considerations in the flavored Higgs sector, i.e., by requiring the total widths of the flavored scalars $Y_{u, d}$ to be small compared to their masses. 

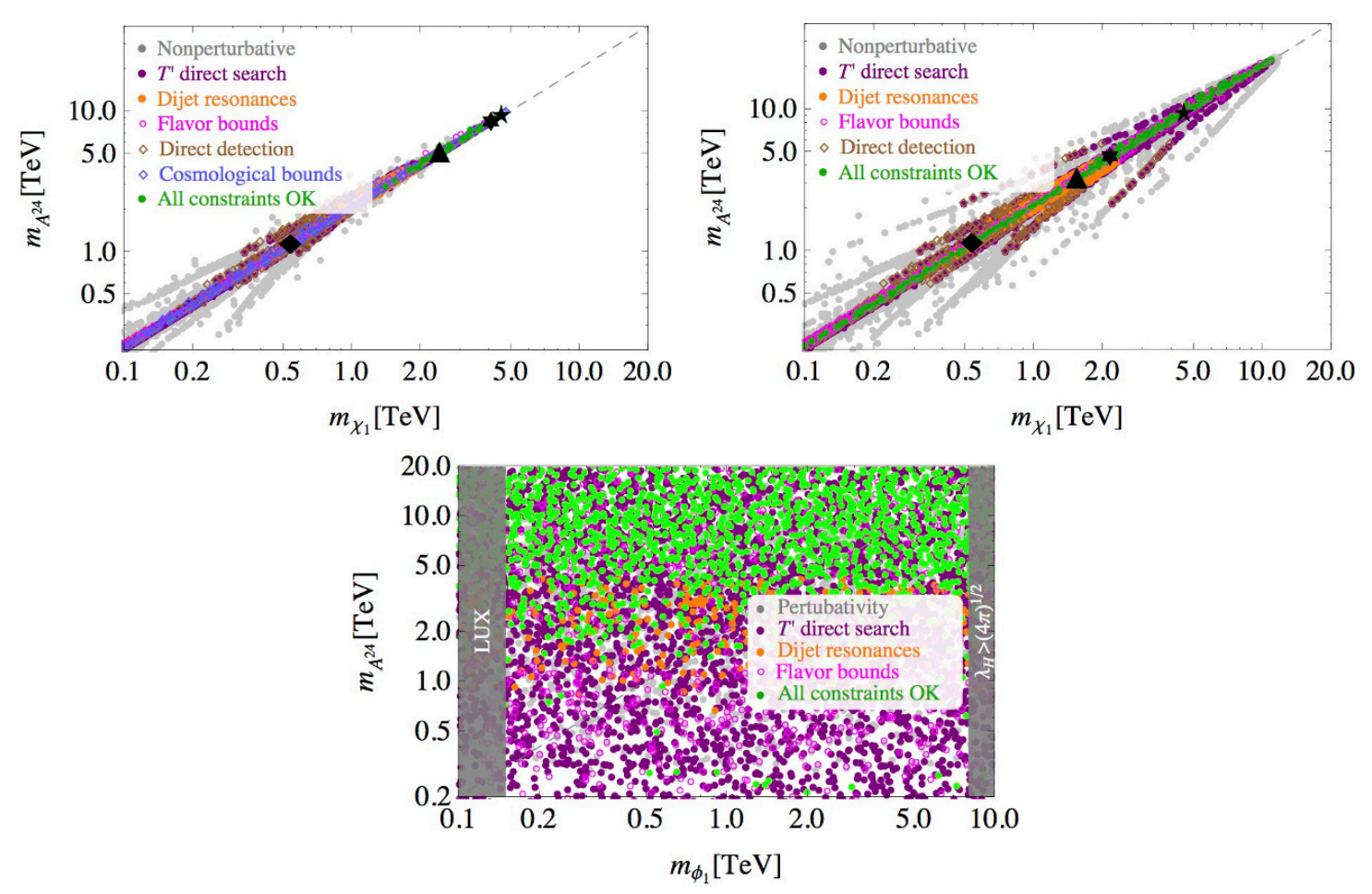

Figure 3. The results of the scan for fermionic DM with radiative mass splitting (upper left panel), in the large mass splitting limit (upper right panel) and scalar (lower panel) flavored DM. Constraints from perturbativity (grey), $t^{\prime}$ (dark magenta) and dijet resonance (orange) searches, flavor bounds (light magenta), early-time cosmology (blue) and direct DM detection (brown) are consecutively applied. Allowed parameter points are denoted by green. For scalar flavor DM (right) we show the LUX and perturbativity bounds as two grey bands. The four benchmark points for fermionic flavored DM are denoted by a diamond, a triangle, a hexagram and a pentagram.

The results of the scan are shown in figures $3,4,5$. Figure 3 (upper panels) show the results of the scan for fermionic DM model with radiative (left) and large tree-level mass splitting (right). Figure 3 (lower panel) instead shows the results of the scan for scalar DM. All the points shown in figure 3 give the correct relic DM abundance, $\Omega_{\mathrm{DM}}$. Different colors denote consecutively applied constraints. The grey points fail the perturbativity requirement, $\Gamma_{A^{m}}<0.5 m_{A^{m}},\left|\Delta m_{i j}\right|<0.5 m_{\chi}$ discussed above. The points in brown are excluded by direct DM detection, the points in dark magenta by $t^{\prime}$ direct searches and the points in orange by dijet resonance searches. The flavor bounds exclude points in light magenta, while cosmological considerations - mainly from big bang nucleosynthesis exclude points in dark blue. The green points are allowed by all constraints. In figures 4 and 5 we also show the points where it is not possible to obtain the correct relic abundance (denoted by light blue), and denote by dark red the points excluded by the combined direct-detection, collider and flavor constraints.

For fermionic DM the observed relic abundance requires resonantly enhanced annihilation through $s$-channel exchange of the lightest FGB, $A^{24}$, see figure 6 (left). This leads to 

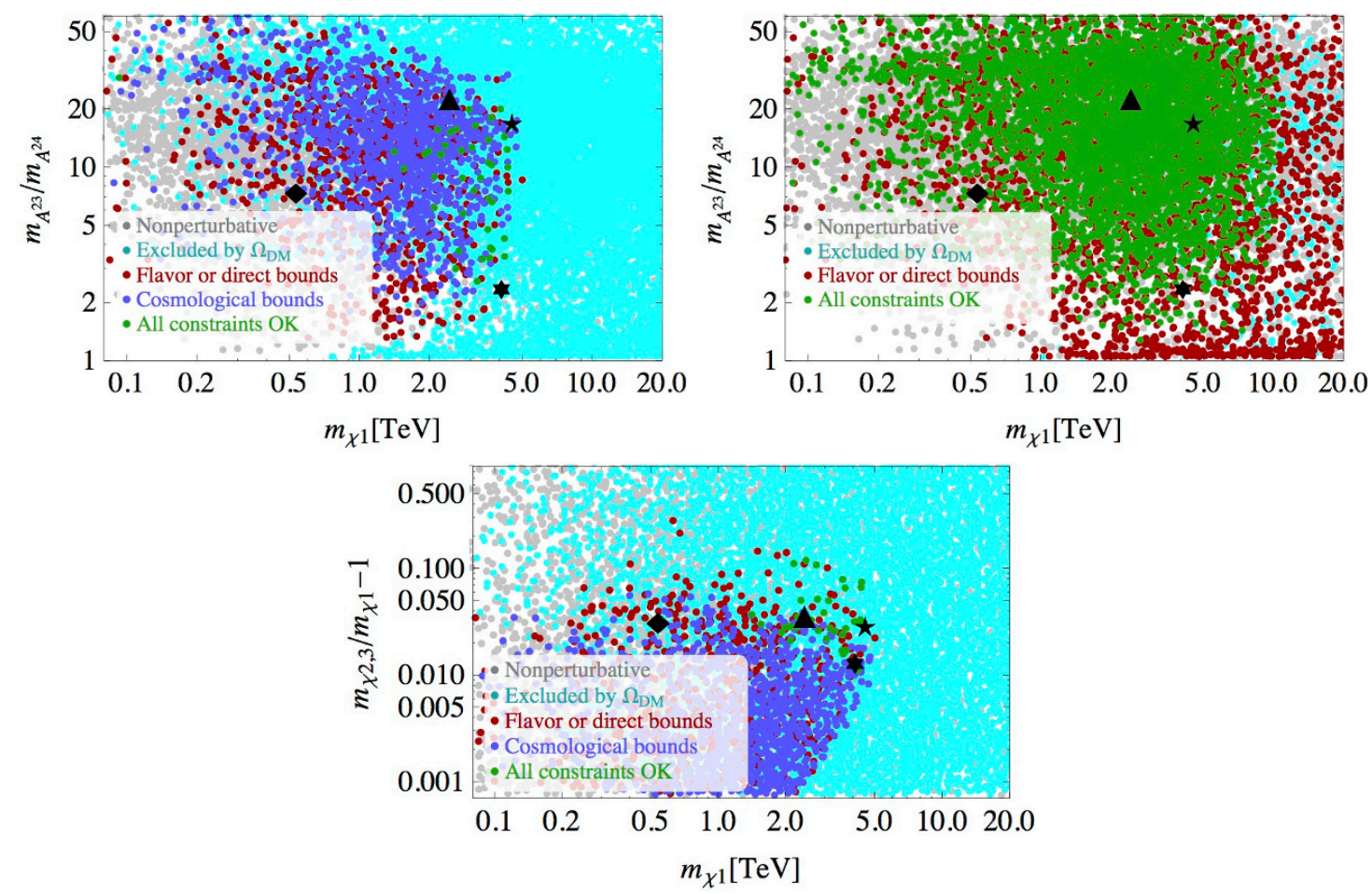

Figure 4. The ratio of masses of the next-to-lightest to the lightest FGBs, $m_{A^{23}} / m_{A^{24}}$ for radiatively split DM multiplet (upper left panel), and for the large mass splitting limit (upper right panel), as functions of the DM mass, $m_{\chi_{1}}$, for the fermionic flavored DM. Lower panel shows the relative radiative mass splitting in the DM multiplet. The constraints due to perturbativity (grey), too large relic abundance (light blue), early cosmology (dark blue), flavor and direct bounds (dark red), are applied consecutively, leaving allowed points (green).

the correlation $m_{\chi_{i}} \simeq m_{A^{24}} / 2$ shown in figure 3 (upper panels). It is possible to obtain the correct relic abundance also if the DM mass is only approximately half of the lightest FGB (points away from the diagonal in figure 3 (upper panels)). These points require at least some of the couplings to be large and are excluded by flavor, collider, direct detection, or perturbativity constraints. For the allowed points the relation $m_{\chi_{i}} \simeq m_{A^{24}} / 2$ is satisfied to within a few decay widths of $A^{24}$, i.e. to within $\mathcal{O}(10 \%)$. The scalar flavored DM, on the other hand, predominantly annihilates through the Higgs portal, see figure 6 (right). There is thus no relation between $m_{\phi}$ and $m_{A^{24}}$, as seen in figure 3 (lower panel).

In the remainder of this section we discuss how the various constraints on the DM model were obtained.

\subsection{Thermal relic}

For the calculation of the DM relic density we follow refs. [31, 32]. To speed-up the numerical scan we work in the non-relativistic limit, using the freeze-out approximation. The details of the calculation are given in appendix B. Among viable parameter points we 

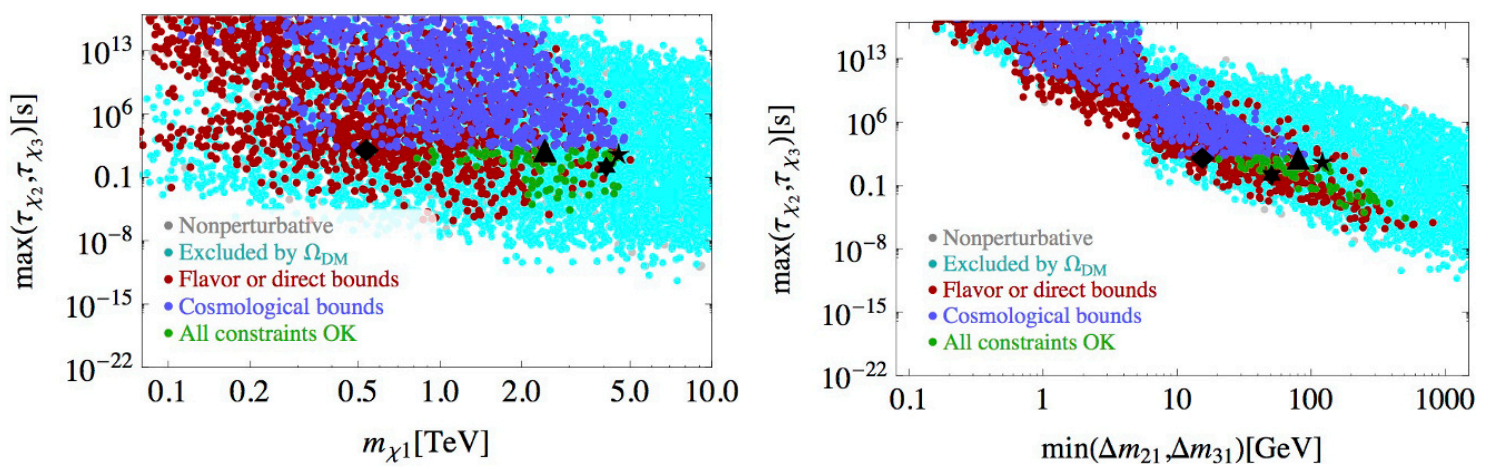

Figure 5. The maximal decay time of the two heavy states in the DM multiplet as functions of DM mass (left) and the minimal mass splitting in the DM multiplet (right) for radiatively split fermionic flavored DM. The color coding is as in figure 4.
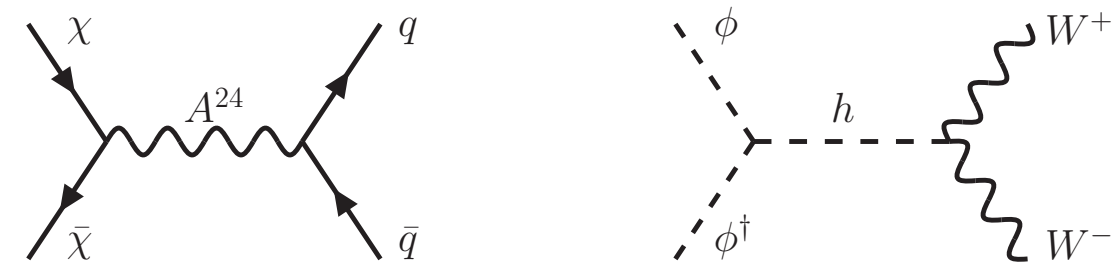

Figure 6. The Feynman diagrams for the dominant processes in the DM annihilation for fermionic (left) and scalar (right) flavored DM. For scalar DM only one representative diagram is shown; other relevant final states include $b \bar{b}, c \bar{c}, \tau \tau$ and $t \bar{t}, h h, Z Z$ (when kinematically allowed).

choose four benchmarks that satisfy all other experimental constraints. For the benchmark points we verify the DM relic abundance calculation using the MadDM [33] package. We computed the required Feynman rules using the Feynrules [34] package.

\subsubsection{Fermionic dark matter}

In the fermionic DM model the DM annihilation to quarks is dominated by $s$-channel exchange of the lightest FGB, $A^{24}$, see figure 6 (left panel). The $\chi_{i} \bar{\chi}_{i} \rightarrow \bar{u}_{j} u_{j}$ annihilation cross section is given by

$$
\sigma\left(\chi_{i} \bar{\chi}_{i} \rightarrow \bar{u}_{j} u_{j}\right) \simeq \frac{\left(\hat{g}_{\chi}^{24}\right)_{i i}^{2}}{4 \pi} \frac{s^{1 / 2}\left(s+2 m_{\chi_{i}}^{2}\right)}{\sqrt{s-4 m_{\chi_{i}}^{2}}} \frac{\left(\hat{\mathcal{G}}_{V}^{u}\right)_{j j, 24}^{2}+\left(\hat{\mathcal{G}}_{A}^{u}\right)_{j j, 24}^{2}}{\left(s-m_{A^{24}}^{2}\right)^{2}+m_{A^{24}}^{2} \Gamma_{A^{24}}^{2}},
$$

where

$$
\hat{\mathcal{G}}_{V, A}^{u}=\left(\hat{\mathcal{G}}_{L}^{u} \pm \hat{\mathcal{G}}_{R}^{u}\right) / 2,
$$

$\sqrt{s}$ is the center of mass energy and $\Gamma_{A^{24}}, m_{A^{24}}$ are the decay width and mass of the lightest FGB, respectively. In eq. (4.1) we have neglected quark masses; the full expression is given in eq. (B.8). The $\chi_{i} \bar{\chi}_{i} \rightarrow \bar{d}_{j} d_{j}$ annihilation cross section follows from eq. (4.1) by replacing 
$u \rightarrow d$. The full decay width of the lightest FGB is the sum of all partial decay widths for kinematically allowed channels,

$$
\Gamma\left(A^{24} \rightarrow \bar{u}_{j} u_{j}\right) \simeq \frac{m_{A}^{24}}{4 \pi}\left(\left(\hat{\mathcal{G}}_{V}^{u}\right)_{j j, 24}^{2}+\left(\hat{\mathcal{G}}_{A}^{u}\right)_{j j, 24}^{2}\right) .
$$

In the above expression we have neglected the quark masses for simplicity, with the full expression given in eq. (B.9). The rates for $A^{24} \rightarrow \chi_{i} \bar{\chi}_{i}, \bar{d}_{j} d_{j}$ are obtained by trivial coupling replacements and by correcting the color multiplicity factors.

The correct relic abundance requires resonant annihilation ${ }^{1}, m_{\chi} \simeq m_{A^{24}} / 2$, see figure 3 (upper panels). This implies an upper bound on the DM mass through the following argument. The thermally averaged DM annihilation cross section scales in the narrow width approximation as

$$
\langle\sigma v\rangle \propto \frac{g_{A^{24}}^{4}}{m_{A^{24}} \Gamma_{A^{24}}}+\mathcal{O}\left(\Gamma_{A^{24}} / m_{A^{24}}\right) \sim \frac{1}{\langle Y\rangle_{A^{24}}^{2}} .
$$

Here, we used the approximate scaling for the FGB masses and decay widths, $m_{A^{24}} \sim$ $\langle Y\rangle_{A^{24}} g_{A^{24}}, \Gamma_{A^{24}} \sim\left(g_{A^{24}}\right)^{2} m_{A^{24}}$. The $\langle Y\rangle_{A^{24}}$ and $g_{A^{24}}$ are, respectively, the projections of the $Y_{u, d}$ vevs and $g_{Q, U, D}$ couplings onto the lightest FGB, $A^{24}$. The DM relic abundance is $\Omega_{\mathrm{DM}} \propto 1 /\langle\sigma v\rangle \propto\langle Y\rangle_{A^{24}}^{2}$ and thus depends predominantly only on the flavon vevs. Not exceeding the relic abundance puts an upper bound $\langle Y\rangle_{A^{24}} \lesssim \mathcal{O}$ (few $\left.100 \mathrm{GeV}\right)$, almost independent of the DM mass. Since $m_{A^{24}} \sim\langle Y\rangle_{A^{24}} g_{A^{24}}$, and $g_{A^{24}} \lesssim \sqrt{4 \pi}$ for the theory to be perturbative, this also sets an upper bound on the lightest FGB mass. This in turn implies an upper bound on the DM mass through the relation $m_{\chi} \simeq m_{A^{24}} / 2$.

In the limit where only $\chi_{1}$ contributes to the DM relic abundance we find, using the scan, an upper bound $m_{\chi_{1}} \lesssim 10 \mathrm{TeV}$. The approximation is valid if $\chi_{2,3}$ states decay well before $\chi_{1}$ freezes out (i.e. $\tau_{\chi_{2,3}} \lesssim 10^{-11}$ s for $m_{\chi} \sim 1 \mathrm{TeV}$ ). For purely radiative DM mass splitting this is never the case (cf. figure 5). Instead, if $\chi_{2,3}$ decay after decoupling, they will also contribute to the final DM relic abundance and one needs to sum all three contributions. In this case, the constraints on the mass spectrum become much more severe. In particular, in order for all $\chi$ components to annihilate efficiently their masses need to be within a few decay widths away from the lightest FGB (LFGB) mass. This in turn implies that the (radiative) DM mass splitting has to be of the order of the LFGB width. Since the splitting increases with $g_{U}$ we expect these effects to decrease the effective thermal DM annihilation cross section much before the theory becomes non-perturbative. Indeed we find, using the scan, an upper bound $m_{\chi_{1}} \lesssim 5 \mathrm{TeV}$.

Figure 4 (upper panels) shows the ratio of masses of the next-to-lightest and the lightest FGB, $m_{A^{23}} / m_{A^{24}}$, as a function of DM mass $m_{\chi_{1}}$ for radiatively split DM multiplet (left) and in the large mass splitting limit (right). In most of the parameter space satisfying the $\Omega_{\mathrm{DM}}$ constraint $A^{23}$ is much heavier than $A^{24}$ so that the effects of higher resonances are indeed negligible. This justifies the use of only the lightest FGB when calculating the DM density in the scan.

\footnotetext{
${ }^{1}$ That is to say that some fine-tuning is required in order to obtain the correct relic abundance. However, deviations from the resonant annihilation condition are possible if one relaxes the requirement of perturbative couplings.
} 
Figure 4 (lower panel) shows the relative radiative mass splitting $\Delta m_{21} / m_{\chi_{1}}$ and $\Delta m_{31} / m_{\chi_{1}}$ as a function of the DM mass, $m_{\chi_{1}}$ (both splittings are shown in one plot). In most cases the mass splitting is below $\mathcal{O}(10 \%)$ in order for all $\chi$ components to lie close to the resonant condition, as anticipated.

\subsubsection{Scalar dark matter}

For scalar DM the interactions with the visible sector are mainly due to the Higgs-portal operator, $\lambda_{H}\left(\phi^{\dagger} \phi\right)\left(H^{\dagger} H\right)$, in eq. (3.18). The interactions due to the exchanges of FGBs are subleading except for the resonant annihilation regions $m_{\phi_{1}} \simeq m_{A^{24}} / 2$. By adjusting the value of $\lambda_{H}$ one can obtain the correct relic abundance for any mass of $m_{\phi_{1}}$ irrespective of the lightest FGB mass, $m_{A^{24}}$, see figure 3 (bottom panel). Thus, in contrast with the fermionic DM case, the scalar DM does not link the electroweak and the flavor-breaking scales. In the calculation of the thermal relic abundance we include the following annihilation channels: $\phi_{1}^{\dagger} \phi_{1} \rightarrow \bar{b} b, \bar{c} c, \tau^{+} \tau^{-}, W^{+} W^{-}, Z Z$, $h h$ and $\bar{t} t$, see figure 6 . The annihilation cross sections are

$$
\begin{gathered}
\sigma\left(\phi_{1}^{\dagger} \phi_{1} \rightarrow \bar{f} f\right)=\frac{\lambda_{H}^{2} m_{f}^{2} N_{c}\left(1-4 m_{f}^{2} / s\right)^{3 / 2}}{8 \pi \sqrt{1-4 m_{\phi_{1}}^{2} / s}\left[\left(m_{h}^{2}-s\right)^{2}+m_{h}^{2} \Gamma_{h}^{2}\right]}, \\
\sigma\left(\phi_{1}^{\dagger} \phi_{1} \rightarrow V V\right)=\frac{c_{V} \lambda_{H}^{2}}{16 \pi s} \frac{\sqrt{1-4 m_{V}^{2} / s}\left(12 m_{V}^{4}-4 m_{V}^{2} s+s^{2}\right)}{\sqrt{1-4 m_{\phi_{1}}^{2} / s}\left[\left(m_{h}^{2}-s\right)^{2}+m_{h}^{2} \Gamma_{h}^{2}\right]},
\end{gathered}
$$

where $c_{W}=1, c_{Z}=1 / 2$ and

$$
\sigma\left(\phi_{1}^{\dagger} \phi_{1} \rightarrow h h\right)=\frac{\lambda_{H}^{2}}{32 \pi s} \frac{\sqrt{1-4 m_{h}^{2} / s}\left[\left(2 m_{h}^{2}+s\right)^{2}+m_{h}^{2} \Gamma_{h}^{2}\right]}{\sqrt{1-4 m_{\phi_{1}}^{2} / s}\left[\left(m_{h}^{2}-s\right)^{2}+m_{h}^{2} \Gamma_{h}^{2}\right]} .
$$

The thermally averaged cross sections and relic abundances are computed following the prescription described in appendix B. The results of the scan are given in figure 3 (bottom panel). In figure 7 we plot the coupling $\lambda_{H}$ necessary to obtain correct DM relic density as a function of the DM mass, $m_{\phi_{1}}$. As commented in section 3.2 the flavon vevs split the lightest DM state $\phi_{1}$ from the heavier ones, such that only $\phi_{1}$ contributes to $\Omega_{\mathrm{DM}}$ (lower dashed line). Instead, if the splitting is too small for $\phi_{2,3}$ to decay before freeze-out, all three components contribute (upper dashed line). In both cases, requiring the Higgs-portal coupling $\lambda_{H}<\sqrt{4 \pi}$, such that the relic-abundance calculation is well in the perturbative regime, limits the DM mass $m_{\phi_{1}} \lesssim 8 \mathrm{TeV}$.

Note that the role of the Higgs portal may be played by other light scalars. In ref. [21] the flavon field of the Abelian horizontal symmetry was used to enhance the DM annihilation cross section. If the flavons are light, they can also modify the phenomenology of the fermionic flavored DM, allowing DM annihilation into flavons. In this case the DM phenomenology of the fermionic flavored DM would be closer to the one of our scalar DM model. 


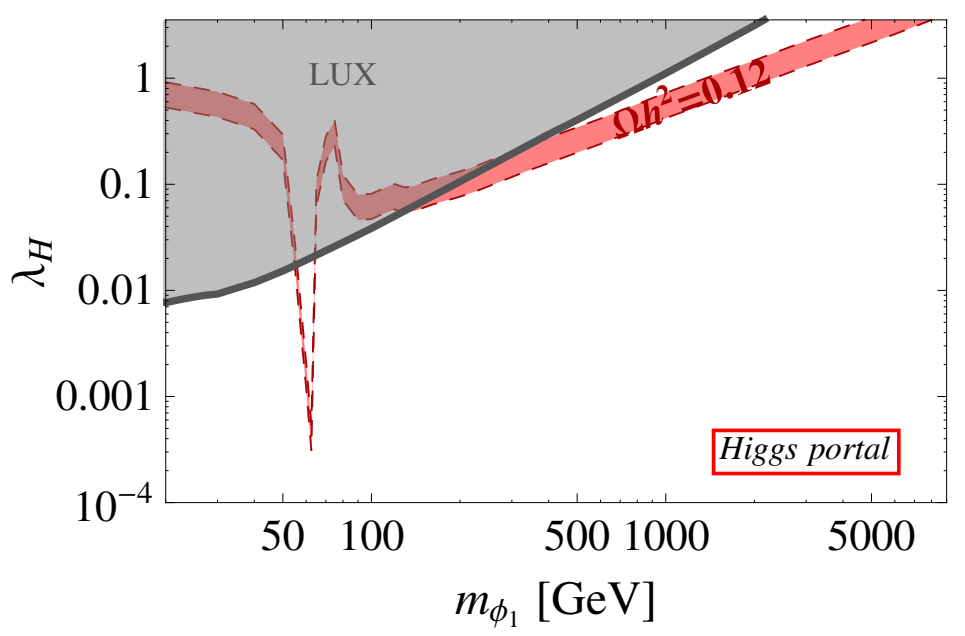

Figure 7. The Higgs-DM coupling, $\lambda_{H}$, as a function of DM mass that gives the correct relic abundance for the Higgs portal scalar DM (red band). The upper (lower) dashed edge corresponds to the limit where $\phi_{2,3}$ decay much after (before) the thermal freeze-out of $\phi_{1}$. The LUX bound, assuming correct relic abundance, is shown as a shaded grey region.

\subsection{Cosmology}

The heavier flavored DM states, both for the fermionic DM, $\chi_{2,3}$, and scalar DM, $\phi_{2,3}$, are unstable. They decay through the $\chi_{i} \rightarrow \chi_{j} \bar{q} q^{\prime}$ transition when the mass splitting is larger than the pion mass, and through the $\chi_{i} \rightarrow \chi_{j} \gamma \gamma$ otherwise, cf. eqs. (3.14), (3.15). The SM particles in the final state of these decays can have various observable effects in cosmology and astrophysics.

The two relevant sets of parameters are the lifetimes of the two heavy states, $\tau_{\chi_{2,3}}$, and the related mass splittings of the DM multiplet (with respect to the lightest state), $\Delta m_{31}, \Delta m_{21}$. The lifetimes determine at which cosmological epoch the heavy states decay. The mass splittings control the released combined electromagnetic and hadronic energy, $E_{\mathrm{vis}} \simeq \Delta m_{21,31}$. They also determine the relic abundances of the heavy states. Generically, near the degenerate limit each state contributes roughly a third of the total DM relic abundance, $\Omega_{\mathrm{DM}}$. Close to the resonant condition $m_{\chi} \simeq m_{A^{24}} / 2$ the $\chi_{1,2,3}$ relic abundances may differ from $\Omega_{\mathrm{DM}} / 3$, depending on the common DM mass and relative mass splittings.

For the scalar DM the mass splitting is expected to be large. The $\phi_{2,3}$ therefore decay before primordial nucleosynthesis. The decays yield negligible entropy release due to the small $\phi_{2,3}$ abundances. Such scenarios are basically unconstrained by current cosmological observations. The same is true for the fermionic DM if additional spurions split the DM multiplet at tree level.

If the fermionic DM multiplet is split solely by radiative corrections, the $\chi_{2,3}$ and $\chi_{1}$ are generically much more degenerate, cf. figure 4 (right). The $\chi_{2,3}$ states are then potentially long lived. For $\tau_{2,3} \sim \mathcal{O}\left(10^{-1} \mathrm{~s}-10^{12} \mathrm{~s}\right)$ the decays may affect the primordial 
generation of light nuclear elements [35]. For longer lifetimes, $\tau_{2,3} \sim \mathcal{O}\left(10^{10} \mathrm{~s}-10^{13} \mathrm{~s}\right)$, the $\chi_{2,3}$ decays distort the thermalization of the CMB by injecting high-energy photons into the plasma before recombination, which is strongly constrained [36, 37]. The $\chi_{2,3}$ states with lifetimes longer than $\tau_{2,3} \gtrsim 10^{10} \mathrm{~s}$ are ruled out, if the injected photons carry energy above the thresholds of the efficient thermalization processes. Typically this is a fraction of the electron mass. For even longer lifetimes, $\tau_{2,3} \gtrsim 10^{13} \mathrm{~s}$, the $\chi_{2,3}$ states decay after recombination. This results in photons that free-stream to us and can be searched for in diffuse galactic and extra-galactic gamma and X-ray spectra. A combination of measurements excludes scenarios with $\tau_{2,3} \lesssim 10^{26}$ s all the way down to $\Delta m_{21,31} \gtrsim$ $\mathcal{O}(10) \mathrm{keV}[38]$.

In the remainder of this section we consider in more detail the region $\tau_{\chi_{2,3}} \sim$ $10^{-1} \mathrm{~s}-10^{12} \mathrm{~s}$, where the big bang nucleosynthesis (BBN) provides the most stringent constraints $[35,39]$. The injection of energetic photons or hadrons from $\chi_{2,3}$ decays during or after BBN adds an additional non-thermal component to the plasma that can modify the abundances of the light elements [40-44]. The bounds differ depending on whether the decays result in hadronic or electromagnetic showers in the plasma. The most stringent bounds are for a relic that produces mostly hadronic showers. This is because the electromagnetically interacting particles such as photons and electrons thermalize very quickly by interacting with the tail of the CMB distribution until the universe is $10^{6} \mathrm{~s}$ old. In our case, the decays $\chi_{2,3} \rightarrow \chi_{1} q \bar{q}^{\prime}$ are always kinematically allowed for $\tau_{\chi_{2,3}}<10^{12} \mathrm{~s}$. The $\chi_{2,3}$ decays thus predominantly produce a small number of hadronic jets with a combined released hadronic energy $E_{\text {had }} \simeq E_{\text {vis }}$.

There are three distinct ranges of lifetimes [45]. For $0.1 \mathrm{~s} \lesssim \tau_{\chi_{2,3}} \lesssim 100 \mathrm{~s}$ the dominant effect is the inter-conversion between protons and neutrons, which overproduces the ${ }^{4} \mathrm{He}$ abundance. For longer lifetimes, $100 \mathrm{~s} \lesssim \tau_{\chi_{2,3}} \lesssim 10^{7} \mathrm{~s}$, hadro-dissociation is the most efficient process and the bounds come from the non-thermal production of $\mathrm{Li}$ and $\mathrm{D}$. At late times, $10^{7} \mathrm{~s} \lesssim \tau_{\chi_{2,3}} \lesssim 10^{12} \mathrm{~s}$, photo-dissociation caused by direct electromagnetic showers or by electromagnetic showers from daughter hadrons can lead to overproduction of ${ }^{3} \mathrm{He}$.

We impose the ${ }^{4} \mathrm{He}, \mathrm{D}$ and ${ }^{3} \mathrm{He}$ constraints ${ }^{2}$ using the results in ref. [45]. The visible energy release in the decays is $E_{\mathrm{vis}} \sim \Delta m_{21,31}$. For $100 \mathrm{GeV}<\Delta m_{21,31}<10 \mathrm{TeV}$ the constraints derived from the three relic mass benchmarks in ref. [45] are well approximated by a power-law scaling with $E_{\mathrm{vis}}^{-\eta_{i}}$. The exponents for the three constraints are $\eta_{4} \mathrm{He} \approx 1 / 3$, $\eta_{\mathrm{D}} \approx 1 / 2$ and $\eta_{3} \mathrm{He} \approx 1$. For inter-conversion and hadro-dissociation the power-law scaling is expected to break down at energies below $\mathcal{O}(10) \mathrm{GeV}$ due to the presence of hadronic thresholds [45]. We thus do not extrapolate the fit results for ${ }^{4} \mathrm{He}$ and $\mathrm{D}$ for $\Delta m_{21,31}$ below $10 \mathrm{GeV}$. We assume that the photo-dissociation effects retain approximate power law behavior for $E_{\text {vis }}$ large compared to the binding energies of the light nuclei, which is of the order of few tens of $\mathrm{MeV}$. In our model for $\tau_{\chi_{2,3}}<10^{2(12)} \mathrm{s}$, the mass splitting,

\footnotetext{
${ }^{2}$ The measured ${ }^{4} \mathrm{He}$ abundance has shifted upwards significantly since the publicaton of ref. [45]. This should weaken the constraints for $\tau_{2,3} \lesssim 100 \mathrm{~s}$. The upward shift has no consequences for our conclusions since we find that the ${ }^{4} \mathrm{He}$ constraint from ref. [45] is already never important in excluding the viable parameter space in our models.
} 
$\Delta m_{21,31}$, is always above $10(0.1) \mathrm{GeV}$. Our approximations are thus always valid for ranges of lifetimes for which the ${ }^{3} \mathrm{He}$ constraints are the most stringent. For the deuterium bound, on the other hand, the power-law scaling is expected to fail for part of the parameter space where the bound is the most stringent, since $\Delta m_{21,31}$ can be as low as a few GeV. We have checked using the power-law derived bound that these regions are excluded by several orders of magnitude. This gives us confidence to conclude that they remain excluded even with a more faithful treatment of hadro-dissociation effects.

In figure 5 we show the distribution of $\chi_{2,3}$ lifetimes in the viable parameter space of the fermionic DM model. The cosmological constraints rule out all points with $\tau_{2,3} \gtrsim 100 \mathrm{~s}$, which is the range of lifetimes for which the deuterium bound becomes effective. The points with lifetimes $\tau_{2,3} \lesssim 100 \mathrm{~s}$, on the other hand, are never excluded by cosmological constraints. This is the range of lifetimes where the most strigent bound comes from the ${ }^{4} \mathrm{He}$ abundance, which, however, is not sufficient to exclude any of our fermionic DM model points.

\subsection{Direct and indirect dark matter searches}

Both fermionic and scalar flavored DM can produce direct detection signal from DM scattering on nuclei. For fermionic DM the scattering is due to $t$-channel exchanges of FGBs. For scalar DM the scattering is dominated by the Higgs exchange in the $t$-channel, while the contribution of FGBs is in general negligible.

The spin-independent interactions with the nucleons for the fermionic flavored DM are described by the following effective Lagrangian $[46,47]$

$$
\mathcal{L}_{\text {dir. }}=f_{p}\left(\bar{\chi} \gamma_{\mu} \chi\right)\left(\bar{p} \gamma^{\mu} p\right)+f_{n}\left(\bar{\chi} \gamma_{\mu} \chi\right)\left(\bar{n} \gamma^{\mu} n\right)
$$

The Wilson coefficients $f_{p}$ and $f_{n}$ are the couplings to protons and neutrons, respectively,

$$
f_{p}=\sum_{m}\left(\hat{g}_{\chi}^{m}\right)_{11} \frac{2\left(\hat{\mathcal{G}}_{V}^{u}\right)_{11, m}+\left(\hat{\mathcal{G}}_{V}^{d}\right)_{11, m}}{m_{A^{m}}^{2}} \quad \text { and } \quad f_{n}=\sum_{m}\left(\hat{g}_{\chi}^{m}\right)_{11} \frac{\left(\hat{\mathcal{G}}_{V}^{u}\right)_{11, m}+2\left(\hat{\mathcal{G}}_{V}^{d}\right)_{11, m}}{m_{A^{m}}^{2}}
$$

$\hat{\mathcal{G}}_{V}^{u, d}$ are the vectorial couplings of FGBs to quarks, defined in eq. (4.2). The spinindependent DM-nucleon cross section as measured by the LUX experiment [48] is

$$
\sigma_{\chi N}^{\mathrm{SI}}=\left[1+\frac{Z}{A}\left(\frac{f_{p}}{f_{n}}-1\right)\right]^{2} \frac{\mu_{\chi n}^{2} f_{n}^{2}}{\pi}
$$

where $\mu_{\chi n}$ is the reduced mass of the $(\chi, n)$ system. The Xenon atomic and mass numbers are denoted by $Z$ and $A$, respectively. We thus have $Z=54$, while $A$ varies between 128 and 134 . With the above relations we calculate the DM-nucleon cross section and compare it with the current best limits reported by the LUX experiment [48]. The results of the scan are shown in figure 8. Most of the points lie well below the present LUX bound. This is a consequence of the fact that the relic abundance is given by the $s$-channel resonant annihilation, while the direct detection scattering is due to $t$-channel exchanges of FGBs and thus not resonantly enhanced. 

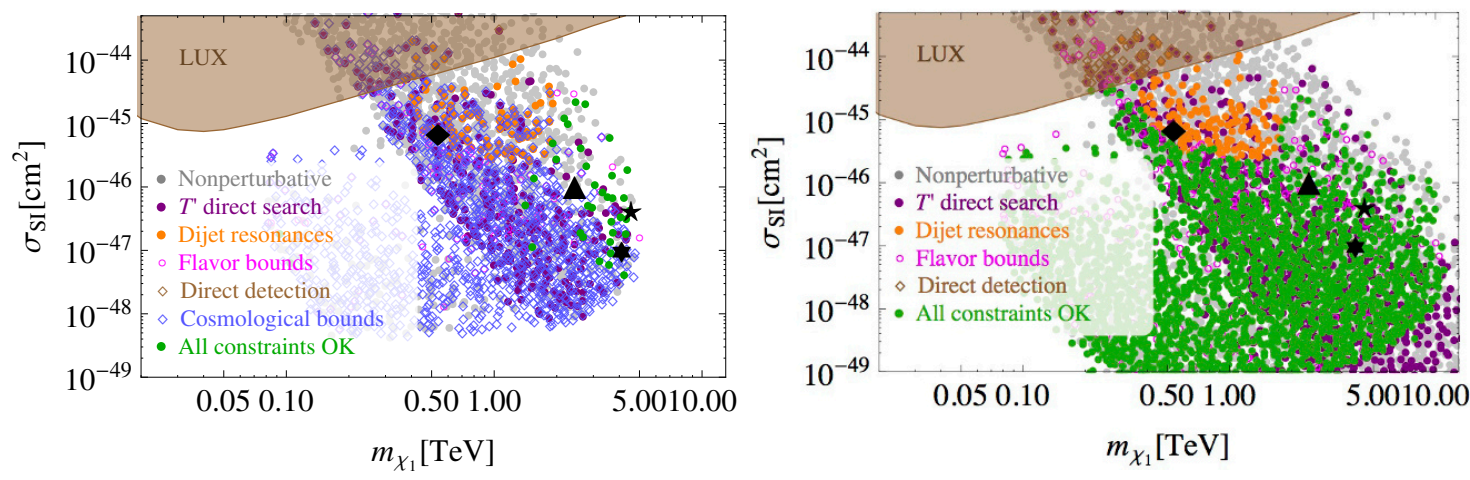

Figure 8. The predicted spin-independent cross section for DM scattering on nuclei as a function of DM mass for radiatively split fermionic DM (left) and in the large mass-splitting limit (right). The LUX bound is the brown shaded region. The color coding for the points is as in figure 3 .

For scalar flavored DM the dominant scattering is through $t$-channel Higgs-boson exchange. This leads to the spin-independent scattering on nucleon $N=n, p,[49,50]$

$$
\sigma_{\chi N}^{\mathrm{SI}}=\frac{\lambda_{H}^{2} f_{N, h}^{2}}{4 \pi}\left(\frac{m_{\phi_{1}} m_{N}}{m_{\phi_{1}}+m_{N}}\right)^{2} \frac{m_{N}^{2}}{m_{h}^{4} m_{\phi_{1}}^{2}} .
$$

The Higgs-nucleon coupling is

$$
f_{N, h}=\frac{2}{9}+\frac{7}{9} \sum_{q} f_{q}^{(N)}
$$

where the sum runs over the light quarks, $q=u, d, s . f_{q}^{(N)}$ are defined by the matrix elements of the light-quark scalar currents, $m_{N} f_{q}^{(N)} \equiv\left\langle N\left|m_{q} \bar{q} q\right| N\right\rangle$. For the $s$ quark we use the lattice determination $f_{s}^{(N)}=0.043 \pm 0.011$ [51]. The matrix elements for $u$ and $d$ quarks depend strongly on $\pi N$-scattering data. A Baryon Chiral Perturbation Theory (B $\chi \mathrm{PT}$ ) analysis of the $\pi N$-scattering data gives $\sigma_{\pi N}=59(7) \mathrm{MeV}$ [52]. This is in agreement with a B $\chi \mathrm{PT}$ fit to world lattice $N_{f}=2+1 \mathrm{QCD}$ data, which gives $\sigma_{\pi N}=52(3)(8) \mathrm{MeV}$ [53]. Including both $\Delta(1232)$ and finite-spacing parametrization in the fit shifts the central value to $\sigma_{\pi N}=44 \mathrm{MeV}$. To be conservative we use $\sigma_{\pi N}=(50 \pm 15) \mathrm{MeV}$ that leads to $f_{u}^{(p)}=$ $(1.8 \pm 0.5) \cdot 10^{-2}, f_{d}^{(p)}=(3.4 \pm 1.1) \cdot 10^{-2}, f_{u}^{(n)}=(1.6 \pm 0.5) \cdot 10^{-2}, f_{d}^{(n)}=(3.8 \pm 1.1) \cdot 10^{-2}$ by using expressions in refs. [54, 55]. This results in $f_{N, h}=(29.7 \pm 1.3) \cdot 10^{-2}$ where we averaged over Higgs couplings to proton and neutron (the difference is an order of magnitude smaller than the quoted error). The resulting bound from the LUX experiment, assuming correct relic abundance, is shown in figure 7 and constrains $m_{\phi_{1}} \gtrsim 150 \mathrm{GeV}$.

Finally, we discuss the constraints from indirect DM searches. The flavored DM annihilates to quarks so that the most constraining indirect DM searches are due to the photon and antiproton cosmic-ray fluxes. The constraints from the antiproton flux are quite dependent on the propagation model. This can lead to almost an order of magnitude difference in uncertainty on the value of the excluded annihilation cross section [56]. 

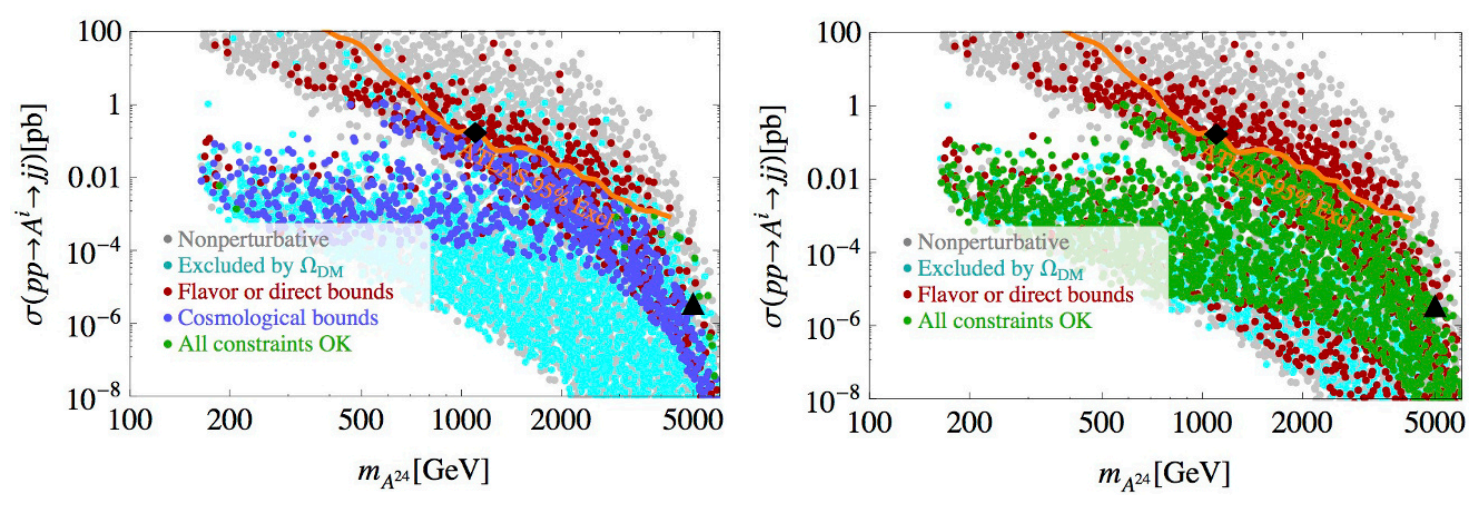

Figure 9. The dijet production cross section at $8 \mathrm{TeV}$ LHC as a function of the lightest FGB mass for radiatively split fermionic DM (left) and in the large mass splitting limit (right). The 95\% CL limit from ref. [61] is denoted with a solid orange line. The color coding is the same as in figure 4 .

For instance, by using the MED propagation model the antiproton-flux measurement by Pamela [57] constrains the DM mass to be $m_{\chi} \gtrsim 20 \mathrm{GeV}$ if the $\chi \chi^{\dagger} \rightarrow b \bar{b}$ annihilation dominates. Similar sensitivity is expected from annihilations to other quarks. The FERMILAT measurements of the photon flux from dwarf spheroidals bound $m_{\chi} \gtrsim 100 \mathrm{GeV}$ for thermal DM annihilating to quarks [58] (there are also slightly less stringent constraints from $\gamma$-ray emissions from the Large Magellanic Cloud [59], and from isotropic $\gamma$-ray background [60]).

\subsection{Searches at the LHC}

The searches for particles beyond the SM at the LHC are sensitive to the lightest new states in our models. The searches for dijet resonances impose constraints on the mass of the lightest FGB [61], and the searches for vector-like $T$ and $B$ quarks impose constraints on the mass of the lightest quark partners $u_{i}^{\prime}, d_{i}^{\prime}$ [62].

The FGBs are narrow resonances that have flavor-conserving as well as flavor-violating couplings to the SM quarks, $u_{i}, d_{i}$, and to the quark partners, $u_{i}^{\prime}, d_{i}^{\prime}$. Since the FGBs are not colored they do not directly couple to gluons. At the partonic level the production process is dominated by $q_{i} \bar{q}_{j} \rightarrow A^{m} \rightarrow q_{k} \bar{q}_{l}$. The FGBs would then appear as resonances in the dijet invariant-mass spectrum. For the most part, the LHC dijet resonance searches are relevant only for the lightest FGB which has, to a very good approximation, flavor-diagonal couplings to quarks. In this case, the cross section for $p p \rightarrow j j$ is given by $[63,64]$

$$
\sigma(p p \rightarrow q \bar{q})=\sum_{i, j} \int_{4 m_{j}^{2}}^{s} \frac{d \mathscr{M}^{2}}{s} \int_{-Y_{B}}^{Y_{B}} d y_{B} \int_{-z_{o}}^{z_{o}} d z f_{i}\left(\sqrt{\tau} e^{y_{B}}\right) f_{\bar{i}}\left(\sqrt{\tau} e^{-y_{B}}\right) \frac{1}{2} \frac{d}{d z} \hat{\sigma}_{i j}
$$


The partonic differential cross section is given by

$$
\begin{aligned}
\frac{d}{d z} \hat{\sigma}_{i j}= & \frac{1}{32 \pi} \beta_{f} \frac{\mathscr{M}^{2}}{\left(\mathscr{M}^{2}-m_{A^{m}}^{2}\right)^{2}+m_{A^{m}}^{2} \Gamma_{A^{m}}^{2}}\left(\left|\hat{\mathcal{G}}_{V}^{u, d}\right|_{i i, m}^{2}+\left|\hat{\mathcal{G}}_{A}^{u, d}\right|_{i i, m}^{2}\right) \\
& \times\left[\left(\left|\hat{\mathcal{G}}_{V}^{u, d}\right|_{j j, m}^{2}+\left|\hat{\mathcal{G}}_{A}^{u, d}\right|_{j j, m}^{2}\right)\left(1+\beta_{f}^{2} z^{2}\right)+4\left(\left|\hat{\mathcal{G}}_{V}^{u, d}\right|_{j j, m}^{2}-\left|\hat{\mathcal{G}}_{A}^{u, d}\right|_{j j, m}^{2}\right) \frac{m_{j}^{2}}{\mathscr{M}^{2}}\right],
\end{aligned}
$$

where, in the partonic center of mass frame, $\mathscr{M}$ is the total energy, $\beta_{f}$ is the velocity of the final-state quarks, $z=\cos \theta^{*}$ is the cosine of the polar angle of the outgoing quark w.r.t. the direction of the incoming quark, and the couplings $\hat{\mathcal{G}}_{V}, \hat{\mathcal{G}}_{A}$ of FGBs to quarks were defined in eq. (4.2). We have only included the $s$-channel contribution that dominates on the FGB resonance peak. Terms odd in $z$ were dropped in the differential cross section since they vanish after integration. The predicted dijet cross sections at the LHC with $\sqrt{s}=8 \mathrm{TeV}$ are shown in figure 9, where the $95 \%$ CL exclusion from ref. [61] is denoted with a solid orange line. This mostly excludes the points where the lightest FGB has large couplings to the quarks. Such points are in fact already mostly excluded either by the perturbativity requirement or from flavor constraints.

The quark partners, $u_{i}^{\prime}, d_{i}^{\prime}$, have an inverted mass hierarchy w.r.t. the SM quarks so that in most of our scan points the $t^{\prime}$ is the lightest state. The bound on the $t^{\prime}$ mass depends on the $t^{\prime} \rightarrow b W, t Z$, and $t h$ branching ratios. The respective partial decay widths are given by

$$
\begin{aligned}
& \Gamma\left(t^{\prime} \rightarrow b W\right)=\frac{g_{w}^{2}}{64 \pi}\left|s_{u_{L} 3} V_{33} c_{d_{L} 3}\right|^{2} \frac{m_{t^{\prime}}^{3}}{m_{W}^{2}}\left(1-x_{W}^{2}\right)^{2}\left(1+2 x_{W}^{2}\right), \\
& \Gamma\left(t^{\prime} \rightarrow t Z\right)=\frac{g_{w}^{2}}{128 \pi}\left(c_{u_{L} 3} s_{u_{L} 3}\right)^{2} \frac{m_{t^{\prime}}^{3}}{m_{W}^{2}} \sqrt{\left[1-\left(x_{Z}+x_{t}\right)^{2}\right]\left[1-\left(x_{Z}-x_{t}\right)^{2}\right]} \\
& \times\left[\left(1-x_{Z}^{2}\right)\left(1+2 x_{Z}^{2}-x_{t}^{2}\right)-x_{t}^{2}\left(1-x_{t}^{2}\right)\right], \\
& \Gamma\left(t^{\prime} \rightarrow t h\right)=\frac{\lambda_{u}^{2}}{64 \pi} m_{t^{\prime}} \sqrt{\left[1-\left(x_{h}+x_{t}\right)^{2}\right]\left[1-\left(x_{h}-x_{t}\right)^{2}\right]} \\
& \times\left[\left(s_{u_{R} 3}^{2} s_{u_{L} 3}^{2}+c_{u_{R} 3}^{2} c_{u_{L} 3}^{2}\right)\left(1+x_{t}^{2}-x_{h}^{2}\right)-4 s_{u_{R} 3} s_{u_{L} 3} c_{u_{R} 3} c_{u_{L} 3} x_{t}\right],
\end{aligned}
$$

where $x_{i}=m_{i} / m_{t^{\prime}}$ and $s_{i}, c_{i}$ are the sines and cosines of the mixing angles between the $\mathrm{SM}$ and exotic quarks, while $V$ is a unitary matrix describing the misalignment of the $Y_{u}$ and $Y_{d}$ vevs. Their definitions can be found in ref. [30], where also the relevant Feynman rules are given. (We present the relevant Higgs Feynman rules in appendix C, correcting an obvious typographical error of ref. [30].) In eq. (4.15) we took the limit $x_{b} \rightarrow 0$ that is justified since $m_{t^{\prime}} \gg m_{b}$. We use the above expressions for the $t^{\prime} \rightarrow b W, t Z$, th branching ratios to obtain the $95 \%$ confidence-level bound on the $t^{\prime}$ mass by interpolating between quoted observed-limits table in [62]. 


\subsection{Flavor constraints}

The model of gauged-flavor symmetries in eq. (3.3) was designed to be compatible with new $\mathrm{TeV}$-scale dynamics and at the same time satisfy the tight flavor constraints from FCNC observables. The FCNCs induced by the exchange of new states are thus relatively mild. The light flavor-violating gauge bosons mediate $\Delta \mathrm{F}=2$ transitions at the tree-level, while the light exotic quarks modify the loop-induced SM process. These modifications are large enough that they restrict the parameter space of the model [30]. All the flavor-violating parameters in the model are fixed by requiring $\left\langle Y_{u}\right\rangle$ and $\left\langle Y_{d}\right\rangle$ to reproduce the observed masses and mixings in the quark sector. The size of the induced FCNCs thus depend only on a relatively small set of flavor conserving parameters in the model, the flavor symmetric masses and couplings. Following the analysis in ref. [30] we focus on $\Delta \mathrm{F}=2$ observables in the neutral $B$ and $K$ sectors, and on $\bar{B}_{s} \rightarrow X_{s} \gamma$.

In our analysis we include the mass differences in the neutral $K^{0}, B_{s}^{0}$, and $B_{d}^{0}$ sectors, $\Delta m_{K}, \Delta m_{B_{d}}$, and $\Delta m_{B_{s}}$, respectively. We also include the indirect $\mathrm{CP}$ violation in the kaon sector, $\varepsilon_{K}$, and the mixing-induced CP asymmetries $S_{\psi K_{s}}$ and $S_{\psi \phi}$. The corresponding mixing amplitude

$$
2 m_{M}\left(M_{12}^{M}\right)^{*}=\left\langle\bar{M}\left|\mathcal{H}_{\mathrm{eff}}^{\Delta M=2}\right| M\right\rangle,
$$

where $M=K^{0}, B_{d}^{0}, B_{s}^{0}$, controls all of these observables.

Two NP contributions to $M_{12}^{M}$ dominate. These are the tree-level exchanges of FGBs and the loop-induced SM-like contribution with internal up-type quarks, including exotic quarks. For the later contribution we first integrate out at the EW scale, $\mu_{W} \simeq m_{W}$, the exotic quarks together with the $W$ and the top quark. In this step we ignore the hierarchy of masses between the exotic quarks and top. This is a good approximation for the dominant contribution that comes from $t^{\prime}$. The theory matches onto the EFT with the SM effective weak operators. We perform the Renormalization Group (RG) of the Wilson coefficients to the low scale at which the hadronic matrix elements are evaluated on the lattice. For the tree-level FGB exchanges the hard scale is given by the corresponding gauge-boson masses. We integrate out the FGB at the corresponding hard scale and RG evolve the Wilson coefficients to the hadronic scale. The FGB exchanges generate four-fermion operators with the Dirac structures that differ from the SM one, namely $\left(\bar{f}_{i} \gamma_{\mu} P_{R} f_{j}\right)\left(\bar{f}_{j} \gamma^{\mu} P_{R} f_{i}\right)$ and $\left(\bar{f}_{i} \gamma_{\mu} P_{R} f_{j}\right)\left(\bar{f}_{j} \gamma^{\mu} P_{L} f_{i}\right)$, where $i, j$ are the flavor indices. The RG evolution is implemented following ref. [65] (for further details and the dependence of the numerical relevance with the scale see also ref. [30]). For the non-perturbative inputs, the decay constants and the bag parameters, we use the current lattice averages [66].

The mass difference in the neutral kaon sector, $\Delta m_{K}$, and the CP-violating parameter $\varepsilon_{K}$ are given by

$$
\Delta M_{K}=2 \operatorname{Re}\left(M_{12}^{K^{0}}\right), \quad \varepsilon_{K}=\frac{\kappa_{\varepsilon} e^{i \varphi_{\varepsilon}}}{\sqrt{2} \Delta M_{K}^{\exp }} \operatorname{Im}\left(M_{12}^{K^{0}}\right)
$$

with $\varphi_{\varepsilon}=(43.51 \pm 0.05)^{\circ}$ and $\kappa_{\varepsilon}=0.923 \pm 0.006$, which includes long-distance effects in both $\operatorname{Im} M_{12}^{K^{0}}$ [67] and in the decay, i.e. $\operatorname{Im} \Gamma_{12}^{K^{0}}$ [68]. Our SM expectation for $\varepsilon_{K}$ incorporates 
the known Next-to-Next-to-Leading-Order (NNLO) QCD corrections due to the charm [69] and charm-top [70] contributions.

The mass differences in the neutral $B$ sectors are given by

$$
\Delta M_{B_{q}}=2\left|M_{12}^{B_{q}}\right|, \quad \text { with } q=d, s .
$$

The CP violation in the neutral $B$ sector is probed by the time-dependent asymmetries in the decays $B_{d}^{0} \rightarrow \psi K_{S}$ and $B_{s}^{0} \rightarrow \psi \phi$ that define the observables

$$
S_{\psi K_{s}}=\sin \left(2 \beta+2 \phi_{B_{d}}\right) \quad \text { and } \quad S_{\psi \phi}=\sin \left(2\left|\beta_{s}\right|+2 \phi_{B_{s}}\right),
$$

respectively. In the conventional parametrization of the CKM matrix the SM phases are given by $V_{t d}^{\mathrm{SM}}=\left|V_{t d}^{\mathrm{SM}}\right| e^{-i \beta}$ and $V_{t s}^{\mathrm{SM}}=-\left|V_{t s}^{\mathrm{SM}}\right| e^{-i \beta_{s}}$. The NP phases are defined through the relation $M_{12}^{B_{q}}=\left|M_{12}^{B_{q}}\right| e^{2 i\left(\beta_{q}+\phi_{B_{q}}\right)}$. The tree level exchanges of FGBs induce such new phases in $\Delta F=2$ matrix elements. These are thus constrained both by the $S_{\psi K_{S}}$ and $S_{\psi \phi}$ asymmetries, and by $\varepsilon_{K}$ in the kaon sector.

The rate of $\bar{B} \rightarrow X_{s} \gamma$ is also modified by the presence of exotic up-type quarks. These can only enhance the $\bar{B} \rightarrow X_{s} \gamma$ rate with respect to the SM expectations [10]. The contributions of FGBs are loop-suppressed. Even though they may be enhanced by $m_{b^{\prime}}$ they are negligible in models with a seesaw-like mass generation for quarks, like the model we consider [71]. The SM prediction for the rate in our analysis includes the known NNLO corrections [72-74].

In our numerical scan we mark parameter space points to have passed the flavor constraints only if the predictions for all our observables lie within three standard deviations of the corresponding experimental values. Whenever theoretical uncertainties are relevant, we include them in quadrature with the experimental ones.

The deviations of the selected FCNC observables from the SM predictions for the four benchmark points are shown in figures 10 to 12 .

\section{Benchmarks}

To illustrate the most relevant phenomenology of fermionic flavored DM we select four representative benchmark points. The main features of the four benchmarks are summarized in figures 10-13. The upper panels in the figures show the spectra for the FGBs, $A^{m}$, the quark partners, $u_{i}^{\prime}, d_{i}^{\prime}$, and the DM multiplet, $\chi_{i}$. Each FGB is represented by four shaded $3 \times 3$ rasters. The shade of the entries in the rasters is approximately logarithmically proportional to the size of the couplings to $u_{R}, d_{R}, u_{L}$ and $d_{L}$, respectively (from left to right). The DM relic abundances as functions of the $\chi_{1}$ mass are shown in the bottom left panels. The lines correspond to our approximate calculation for a radiatively split DM multiplet (red solid line) and for a DM multiplet with large mass splittings (black dashed line). The open diamonds (circles) correspond to the solutions of the coupled Boltzmann equations for the radiative (large) splitting cases which were calculated in MadDM. The approximate and MadDM relic-abundance calculations are in very good agreement for this small subset of benchmarks. In general, however, a disagreement of up $\mathcal{O}(30 \%)$ could be expected due 


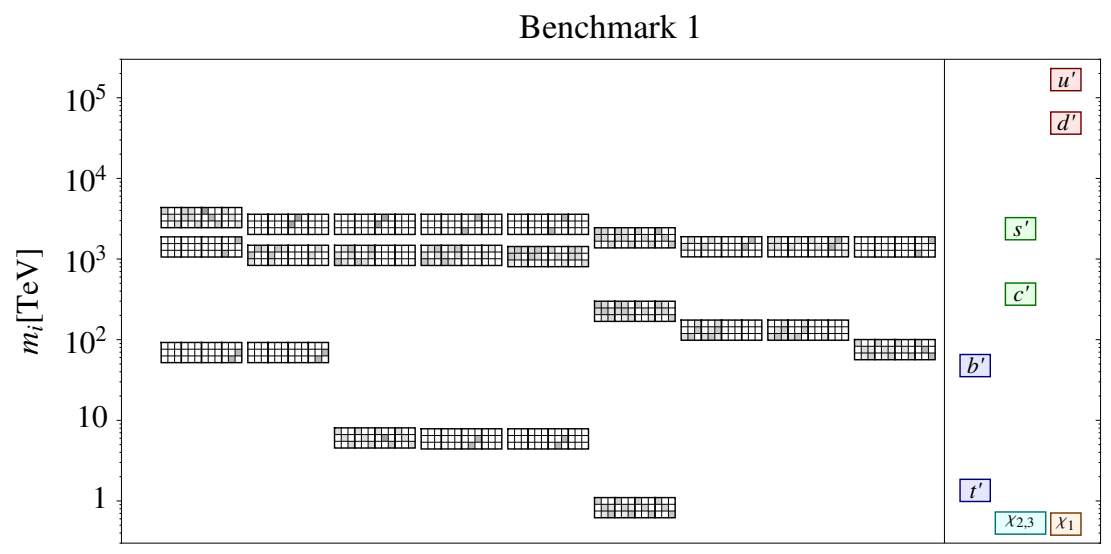

$M_{u}=980 . \mathrm{GeV}, \lambda_{u}=1 ., \lambda_{u}^{\prime}=11.8, M_{d}=700 . \mathrm{GeV}, \lambda_{d}=0.82, \lambda_{d}^{\prime}=3.8, g_{Q}=0.126, g_{U}=0.41, g_{D}=0.237$
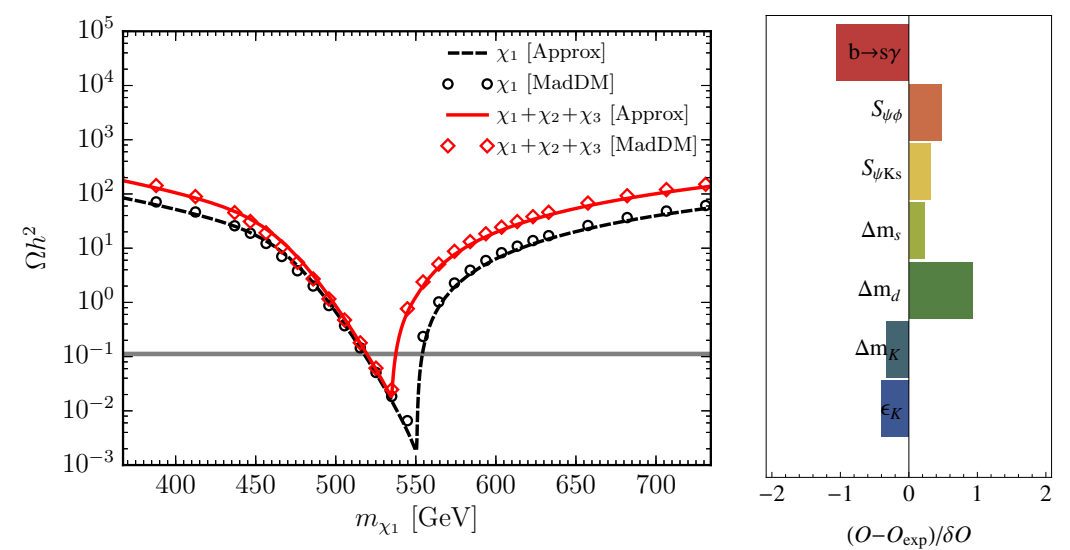

Figure 10. Mass spectrum and flavor decomposition (upper panel), DM relic density as a function of the DM mass with all other parameters fixed (lower left panel) and the pattern of effects in selected flavor observables (lower right panel) for the fermionic flavored DM benchmark 1. The input benchmark-point parameters are listed in the center. See text for details.

to the approximations (see appendix B for a more detailed discussion). The bottom right panels show the pull in selected flavor observables, i.e., the differences between theoretical predictions and measurements normalized to the $1-\sigma$ uncertainties. The uncertainties were obtained by adding in quadrature the theory and experimental errors. The four benchmark points are also marked in figures 3, 4, and 5 with a diamond (1), a five-point star (2), a triangle (3), and a six-point star (4).

"Benchmark 1" is an example of fermionic flavored DM, where the DM multiplet is light, with mass below $1 \mathrm{TeV}$. The mass of the lightest state in the DM multiplet is $m_{\chi_{1}} \simeq 520(540) \mathrm{GeV}$ if it lies just below (above) the LFGB resonance. If the mass splitting between $\chi_{1}, \chi_{2}$ and $\chi_{3}$ is solely due to radiative corrections, $\chi_{2}$ and $\chi_{3}$ are almost mass degenerate with masses roughly $10 \mathrm{GeV}$ above $m_{\chi_{1}}$, and $\chi_{3}$ is about $100 \mathrm{MeV}$ heavier than $\chi_{2}$. The lightest quark partner is $t^{\prime}$ with a mass $m_{t^{\prime}} \simeq 1.3 \mathrm{TeV}$. The lightest FGB has a mass $m_{A^{24}} \simeq 1.1 \mathrm{TeV}$. All the remaining NP states are above $7 \mathrm{TeV}$. This benchmark point 
demonstrates that even parameter regions with low lying FGBs can be consistent with both the resonance searches and the FCNC bounds. The most robust constraints in this parameter region come from cosmology (in case of radiatively-split DM masses) and dijetresonance searches (see figures 5 and 9). Note in particular that for the completely (mass) decoupled fermionic DM scenario, in which cosmology bounds are absent, all experimental constraints can be satisfied even for DM (and LFGB) masses below few $100 \mathrm{GeV}$.

The bottom left panel in figure 10 shows the predicted relic abundance for this benchmark, if only the DM mass is varied, which also modifies the splitting within the DM triplet. Relic abundance consistent with observations is obtained for a mass of DM close to half of the mass of the lightest FGB, in which case the annihilation cross section is resonantly enhanced. To saturate the observed DM relic density, two solutions for $m_{\chi_{1}}$ are obtained, with $m_{\chi_{1}}$ either above or below the resonant peak. We see that for radiatively split DM masses, where all $\chi_{i}$ components contribute to the DM relic density, $m_{\chi_{i}}$ need to lie within $\mathcal{O}(3 \%)$ of the resonant peak for the annihilation to be strong enough. For completely decoupled DM multiplet the resonant condition is relaxed and needs to be satisfied to $\mathcal{O}(5 \%)$.

In figure 10 (upper panel) we show the spectrum for the lower mass solution and radiative DM multiplet splitting. We see that the quark partners of the lighter generations are heavier than the partners of the third generation quarks. Similarly, the FGBs that couple more strongly to the first two generations are typically heavier than the ones that couple preferably to the third generation. The couplings of the lightest FGB to the light quarks have the form $\hat{\mathcal{G}}_{L}^{u} \simeq \hat{\mathcal{G}}_{R}^{u} \simeq \hat{\mathcal{G}}_{L}^{d} \simeq \hat{\mathcal{G}}_{R}^{d} \propto \lambda^{8}$, where the relative corrections to this relation are below the percent level. This means that the lightest FGB couples to the light quarks vectorially, $\hat{\mathcal{G}}_{A}^{u, d} \ll \hat{\mathcal{G}}_{V}^{u, d}$, to a very good approximation. The same is true for the majority of parameter-space points passing flavor constraints.

The largest effects in flavor physics are in the mixing observables, the mass splittings $\Delta m_{d, s}$ in $B_{d}-\bar{B}_{d}$ and $B_{s}-\bar{B}_{s}$ systems, respectively, and the mass splitting in the $K-\bar{K}$ mixing, $\Delta m_{K}$, and the related CP violating parameter $\epsilon_{K}$. The pulls in $b \rightarrow s \gamma$ and $B_{d}-\bar{B}_{d}$ mixing are due to the fact that the measurements agree with the SM prediction only at 1- $\sigma$ level and the contribution to them from new states is very small.

"Benchmark 2" is an example of a generic parameter-space region, but towards the upper end of the perturbatively allowed region. The DM has a mass $m_{\chi_{1}} \simeq 4.5 \mathrm{TeV}$, while the heavier states in the DM multiplet have masses $120 \mathrm{GeV}$ and $150 \mathrm{GeV}$ above $m_{\chi_{1}}$ (for the case of only radiative mass splitting). The lightest exotic quark is the top partner with mass $m_{t^{\prime}} \simeq 1.7 \mathrm{TeV}$, while the mass of the lightest FGB is $m_{A^{24}} \simeq 9.2 \mathrm{TeV}$.

For such high DM masses it is barely possible to obtain the correct relic abundance (see the lower left panel in figure 11). Therefore, the DM mass is finely tuned to be exactly on the resonant peak (see the lower left panel in figure 11), so $m_{\chi_{i}} \simeq m_{A^{24}} / 2$. Because of the high masses of the NP states the direct searches (direct DM detection, $t^{\prime}$ searches and dijet resonance searches) as well as the indirect flavor constraints are easily avoided, although $K-\bar{K}$ mixing does receive non-negligible contributions.

Also in this case, the couplings of the lightest FGB to the light quarks have the form $\hat{\mathcal{G}}_{L}^{u} \simeq \hat{\mathcal{G}}_{R}^{u} \simeq \hat{\mathcal{G}}_{L}^{d} \simeq \hat{\mathcal{G}}_{R}^{d} \propto \lambda^{8}$, so the couplings of the lightest FGB to quarks are to a good extent vectorial. 


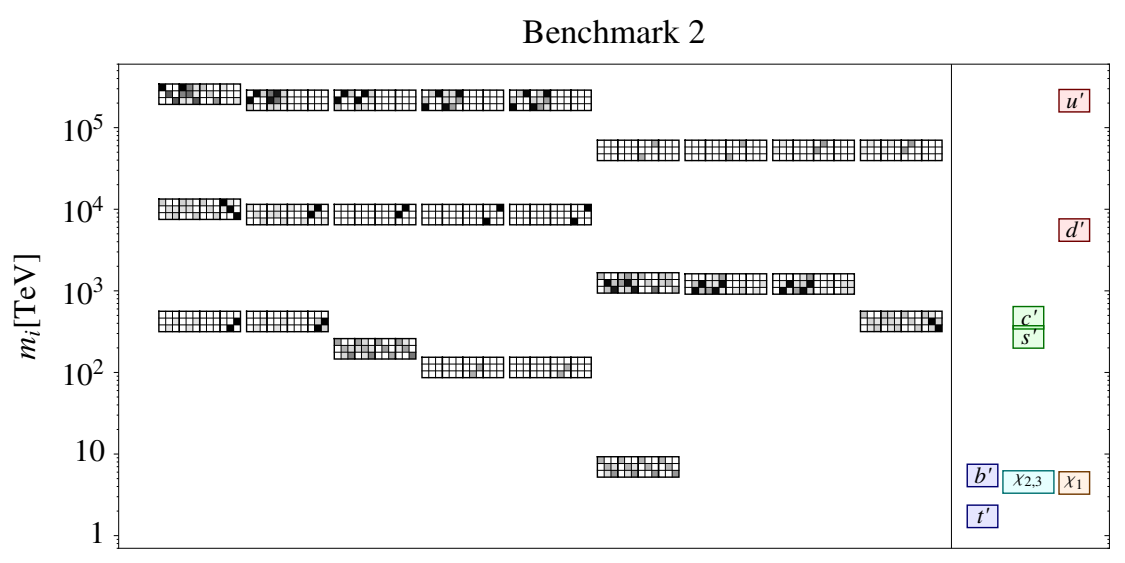

$M_{u}=980 . \mathrm{GeV}, \lambda_{u}=1 ., \lambda_{u}^{\prime}=0.79, M_{d}=360 . \mathrm{GeV}, \lambda_{d}=0.146, \lambda_{d}^{\prime}=0.91, g_{Q}=1.93, g_{U}=0.47, g_{D}=3.4$
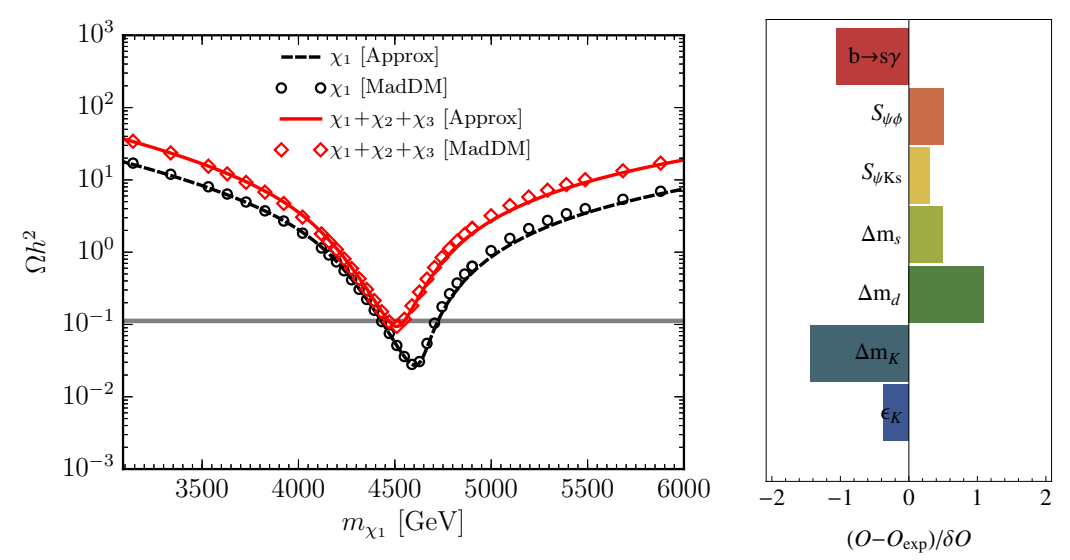

Figure 11. Same as figure 10 for benchmark 2.

"Benchmark 3" is an example of a generic parameter space in which all the couplings of the model are well below the pertubativity bounds. The lightest FGB has a mass $m_{A^{24}} \simeq 5 \mathrm{TeV}$ while all other FGBs have masses above $100 \mathrm{TeV}$. The lightest partner quark is $t^{\prime}$ with mass $m_{t^{\prime}} \simeq 2 \mathrm{TeV}$. The DM states have masses $m_{\chi_{1}} \simeq 2.4 \mathrm{TeV}$ and $m_{\chi_{2}} \simeq m_{\chi_{3}}=2.5 \mathrm{TeV}$ (for radiative mass splitting). All direct experimental constraints as well indirect flavor bounds are easily satisfied in this case. For radiatively DM mass splitting the cosmological constraints are the most constraining. In particular, requiring small enough $\tau_{\chi_{2,3}}$ (or equivalently large enough $\Delta m_{21,31}$ ) typically imposes a lower bound on $g_{U}$.

"Benchmark 4" is an example of the case in which the next-to-lightest FGBs have masses not too far from the lightest FGB. In the benchmark point the lightest FGB has a mass $m_{A^{24}} \simeq 8.3 \mathrm{TeV}$, while the next to lightest FGBs have masses $m_{A^{23}} \simeq m_{A^{22}} \simeq 19 \mathrm{TeV}$, $m_{A^{21}} \simeq 39 \mathrm{TeV}$. In this case the deviations from the $\hat{\mathcal{G}}_{L}^{u} \simeq \hat{\mathcal{G}}_{R}^{u} \simeq \hat{\mathcal{G}}_{L}^{d} \simeq \hat{\mathcal{G}}_{R}^{d} \propto \lambda^{8}$ relation for the lightest FGB coupling to quarks are of $\mathcal{O}(10 \%)$. Nonetheless, this does not have a significant effect on the computation of the DM relic abundance. This is demonstrated in figure 13 bottom left panel where a comparison is shown between the relic-abundance 


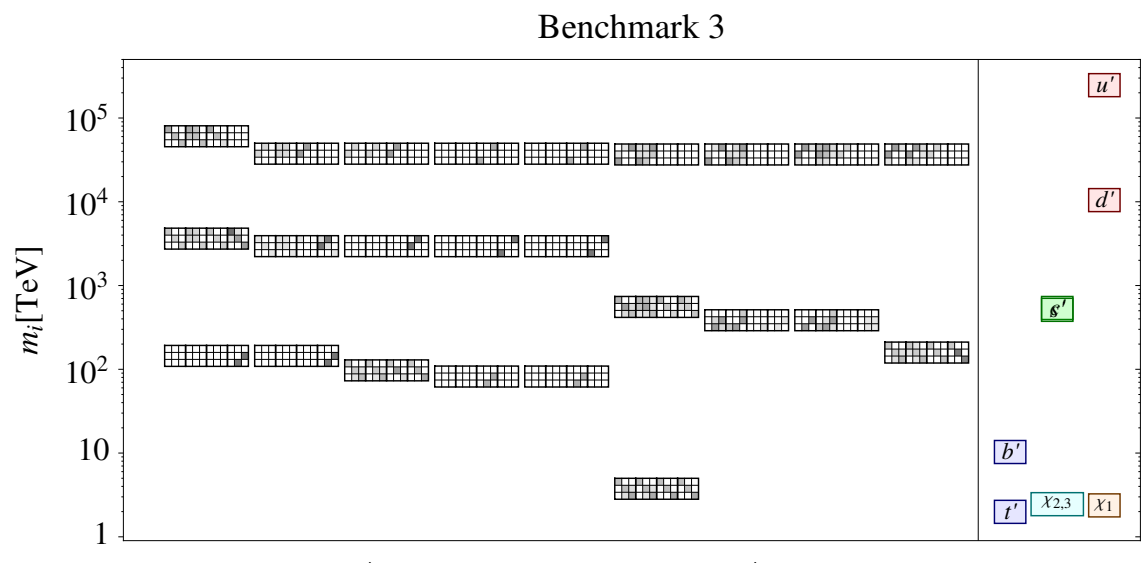

$M_{u}=1210 . \mathrm{GeV}, \lambda_{u}=1 ., \lambda_{u}^{\prime}=1.3, M_{d}=293 . \mathrm{GeV}, \lambda_{d}=0.36, \lambda_{d}^{\prime}=1.24, g_{Q}=0.45, g_{U}=0.46, g_{D}=0.82$
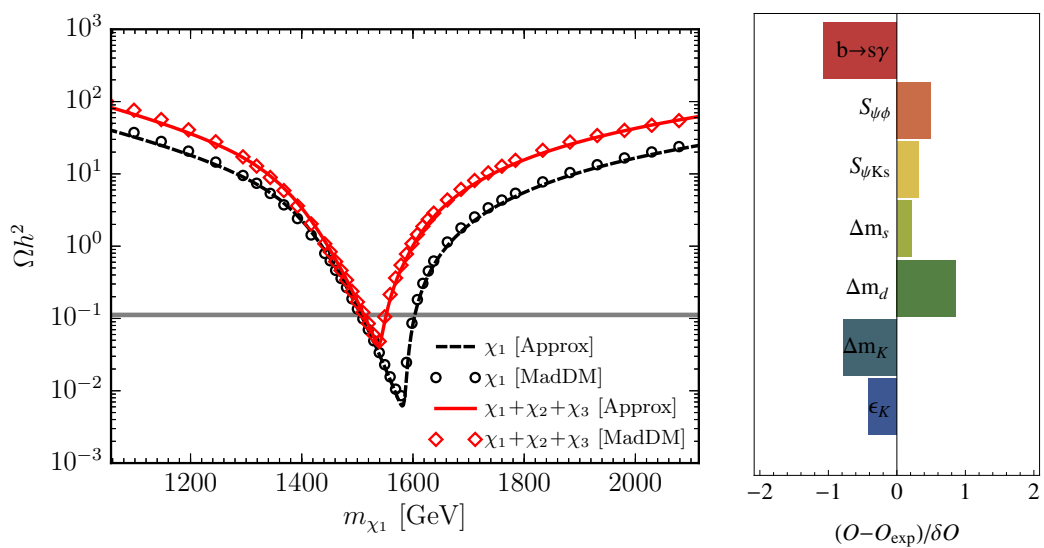

Figure 12. Same as figure 10 for benchmark 3.

calculation neglecting (dashed lines, labelled "Approx") and including (full lines, using MadDM) flavor off-diagonal lightest FGB couplings and contributions of heavier FGBs. For a more detailed discussion of these effects see appendix B.

The lightest quark partner is $t^{\prime}$ with mass $m_{t^{\prime}} \simeq 1.4 \mathrm{TeV}$, and is significantly lighter than all FGBs and also DM. The DM states are degenerate to a good approximation, with masses $m_{\chi_{1}}=4.1 \mathrm{TeV}$ and $m_{\chi_{2}} \simeq m_{\chi_{3}} \simeq 4.2 \mathrm{TeV}$. Because of the light $t^{\prime}$ the flavor constraints are nontrivial, and there are visible effects in $B_{d}$ and $B_{s}$ mixing observables.

\section{Conclusions}

We investigated the possibility that DM is in a nontrivial representation of the continuous flavor group $\mathcal{G}_{F}^{\mathrm{SM}}=\mathrm{SU}(3)_{Q} \times \mathrm{SU}(3)_{U} \times \mathrm{SU}(3)_{D}$. The two main results are that (i) one can have a viable model of DM where DM is stable because it is charged under $\mathcal{Z}_{3}^{\chi}-$ a discrete central subgroup of $\mathcal{G}_{F}^{\mathrm{SM}}$ and color $\mathrm{SU}(3)$, and (ii) that the DM spectrum can be very non-MFV like, while all the low-energy constraints will appear MFV-like. 


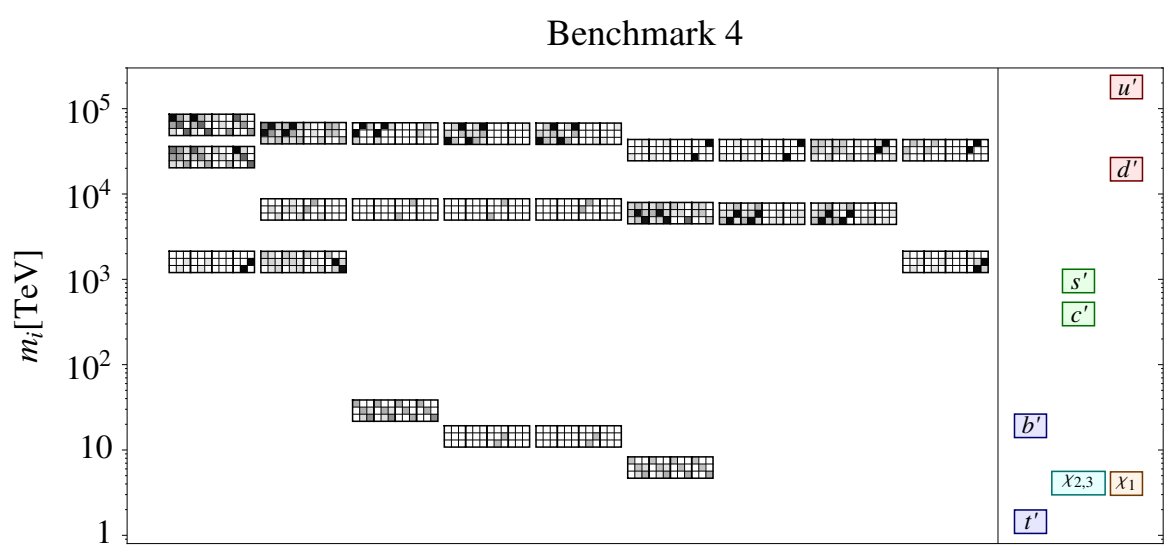

$M_{u}=830 . \mathrm{GeV}, \lambda_{u}=1 ., \lambda_{u}^{\prime}=3.5, M_{d}=1920 . \mathrm{GeV}, \lambda_{d}=0.096, \lambda_{d}^{\prime}=0.48, g_{Q}=1.89, g_{U}=0.312, g_{D}=1.93$
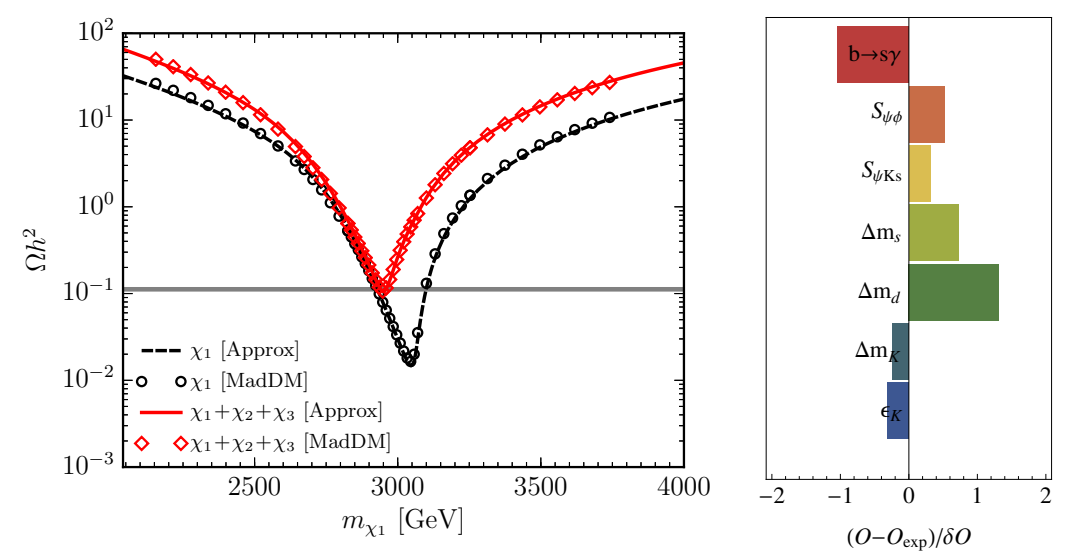

Figure 13. Same as figure 10 for benchmark 4 .

$\mathcal{Z}_{3}^{\chi}$ is exactly conserved in many models of flavor. For instance, it remains unbroken for MFV new physics. More generally, $\mathcal{Z}_{3}^{\chi}$ remains exact if the flavor group $\mathcal{G}_{F}^{\mathrm{SM}}$ is broken only by the vevs of scalar fields, or condensates, with zero flavor triality. Examples of zero flavor triality fields are scalars in bifundamental or in adjoint representations of the flavor $\mathrm{SU}(3)$ 's. The basic requirement for this set-up is that $\mathcal{G}_{F}^{\mathrm{SM}}$ is a good symmetry in the UV. This is achieved, if $\mathcal{G}_{F}^{\mathrm{SM}}$ is fully gauged in the UV, which is the possibility we explored. The DM is then stable because it is $\mathcal{Z}_{3}^{\chi}$ odd, while all SM fields are $\mathcal{Z}_{3}^{\chi}$ even.

We investigated two different types of flavored DM models: (i) models in which the leading interaction of the DM with the visible sector is through the flavored gauge bosons (FGBs), and (ii) models in which the contributions from the FGB exchanges are subleading.

As an example of the first type of models we considered a Dirac fermion DM that is in a fundamental representation of $\mathrm{SU}(3)_{U}$. The relic abundance is fixed by the resonant DM annihilation to SM particles through the $s$-channel exchange of the lightest FGB. The DM is thus required to have a mass of about half of the lightest FGB's mass. This in turn implies that the FGBs cannot be arbitrarily heavy, but at most $\mathcal{O}(10 \mathrm{TeV})$. Such light FGBs are possible, if the masses of FGBs are inversely proportional to the corresponding quark 
masses. That is, if the FGBs that couple most strongly to light quarks are also the heaviest. To achieve this we adopted the model of ref. [10] in which the inverse proportionality is achieved by introducing a set of quark partners also necessary to cancel gauge anomalies. The same quark partners also mix with the SM quarks and lead to the mass hierarchy of the SM quark masses.

The flavor and collider phenomenology of the model is very similar to the case where $\mathrm{DM}$ is not considered. The fact that the first-generation quark partners are the heaviest and that the spectrum is completely split, signals the non-MFV character of the model. However, the low-energy consequences are MFV-like (see appendix A). The flavor constraints are satisfied even with FGBs and the top-quark partner, with masses potentially well below the $\mathrm{TeV}$ scale. The relevant direct collider searches are the searches for dijet resonances and $t^{\prime}$ searches. They exclude part of the available parameter space. Requiring that there is a thermal relic DM introduces new constraints. Because DM is part of a flavor multiplet the heavier DM states need to decay before big bang nucleosynthesis. In the case of radiatively-split fermionic DM this excludes a large part of the parameter space. The remaining points are mostly safe from direct-detection bounds. The fact that the DM mass is related to the FGB mass by the requirement of almost resonant annihilation sets both lower and upper bounds on the DM mass. Requiring that the theory is perturbative also puts an upper bound on the DM mass, $m_{\chi_{1}} \lesssim 5 \mathrm{TeV}$. On the other hand, requiring that the FGBs satisfy flavor and direct constraints and that DM is simultaneously in accordance with cosmological constraints leads to a lower bound on the DM mass, $m_{\chi_{1}} \gtrsim 500 \mathrm{GeV}$. Improved bounds on dijet resonances at the LHC are expected to further strengthen this constraint (see figure 9).

We have also considered the possibility that the DM multiplet is split due to an extra source of flavor breaking. Also in this case, the correct relic abundance requires resonant annihilation. The DM mass is in thus still roughly equal to half of the mass of the lightest FGB. However, the heavier DM states decay well before big bang nucleosynthesis so that a much wider range of DM masses is phenomenologically viable. In our scan this includes DM masses as light as $100 \mathrm{GeV}$ (with very small couplings to FGBs) and up to $10 \mathrm{TeV}$.

A possible signal of the gauged flavor model with fermionic DM at the LHC are monojets, where the lightest FGB is produced associated with initial state radiation and decays to $\chi_{1}$ pairs. The $\chi_{1}$ s are expected to be non-relativistic in the lightest FGB's rest frame, as their combined mass needs to be close to the FGB mass to fulfill the resonance condition for relic DM abundance. In the event that such a signal would eventually emerge, the corresponding dijet-resonance signal is generically also expected in the model. A final possibility in the case of radiatively split DM mass spectrum is that some of the lightest FGBs decay to slow-moving $\chi_{2,3}$. They in turn decay within the detector, leaving (highly) displaced vertices, isolated hits in the calorimeter or in the muon chambers. Unfortunately, in most of the parameter space $\chi_{2,3}$ are expected to decay well outside the detectors, see figure 5, leaving mono-jets as the only signal.

In the second type of models, where FGB exchanges give only subleading contributions, the only visible consequence of the flavor dynamics on the DM is that DM is stable. The DM mass and the mass of the lightest FGB are no longer connected. We show this in the 
example of scalar flavored DM, in which the dominant interactions with the visible sector are through the Higgs portal operator. In this case the phenomenology of the DM is to a very good approximation the same as in the Higgs-portal scalar DM, while the dynamics of FGBs and quark partners is unrelated to DM.

In short, we have shown, using an explicit renormalizable model, that it is possible for flavored DM to be a thermal relic. The considered model is not the only choice. One could consider DM in other representations of $\mathcal{G}_{F}^{\mathrm{SM}}$. Our analysis can be extended also in other ways: for instance, by enlarging the global symmetry as in ref. [14] and subsequently gauging it. For instance, with our field content the global group is $\mathcal{G}_{F}^{\mathrm{SM}} \times \mathrm{SU}(3)_{\chi}$, where $\chi$ is in the fundamental of $\mathrm{SU}(3)_{\chi}$. In our work we have identified $\mathrm{SU}(3)_{\chi}$ with $\mathrm{SU}(3)_{U}$, but other choices could be made. Yet another possibility is to gauge only part of $\mathcal{G}_{F}^{\mathrm{SM}}$, for instance a $\mathrm{U}(2)^{3} \subset \mathcal{G}_{F}^{\mathrm{SM}}$. Note that for fermionic DM, $\mathcal{Z}_{3}$ is part of an accidental $\mathrm{U}(1)_{\chi}$ acting in the dark sector. The $\mathrm{U}(1)_{\chi}$ can be broken by the dimension- 7 operator $L H \chi \chi \chi$, but is exact in our renormalizable model. It can in principle be gauged and lead to additional phenomenology. If $\mathrm{DM}$ is a scalar, $\mathrm{U}(1)_{\chi}$ can be broken already at the renormalizable level, leaving only $\mathcal{Z}_{3}$ exact.

\section{Acknowledgments}

We thank Csaba Csaki, Gino Isidori, Graham Kribs and Christopher Smith for enlightening discussions. J.Z. and F.B. are supported by the U.S. National Science Foundation under CAREER Grant PHY-1151392. This work was supported in part by the Slovenian Research Agency. F.B. is grateful for the hospitality of the Fermilab theory department. Fermilab is operated by Fermi Research Alliance, LLC under Contract No. DE-AC02-07CH11359 with the United States Department of Energy. The work of DR is supported by the NSF under grant No. PHY-1002399.

\section{A Minimal flavor violation with gauged flavor symmetries}

In this appendix we verify numerically that the Wilson coefficients in the weak Hamiltonian for $B_{d}$ and $B_{s}$ mixing,

$$
\mathcal{H}_{\mathrm{eff}}^{\Delta B=2}=\sum_{i=1}^{5} C_{i}^{b q} Q_{i}^{b q}+\sum_{i=1}^{5} \tilde{C}_{i}^{b q} \tilde{Q}_{i}^{b q},
$$

generated from exchanges of flavored gauge bosons, are of the MFV type. A tree-level exchange of FGBs generates contributions to $B_{d}$ mixing through operators

$$
Q_{1}^{b d}=\bar{d}_{L}^{\alpha} \gamma_{\mu} b_{L}^{\alpha} \bar{d}_{L}^{\beta} \gamma_{\mu} b_{L}^{\beta}, \quad Q_{3}^{b d}=\bar{d}_{R}^{\alpha} b_{L}^{\beta} \bar{d}_{R}^{\beta} b_{L}^{\alpha},
$$

and $\tilde{Q}_{1}^{b d}, \tilde{Q}_{3}^{b d}$, that follow from $Q_{1}^{b d}, Q_{3}^{b d}$ with $P_{L} \leftrightarrow P_{R}$ exchange (the remaining operators can be found in, e.g., ref. [75]). If the lightest FGB has predominantly left-handed couplings, then the $C_{1}^{b d}$ Wilson coefficient is the largest one. If the lightest FGB couples predominantly to the right-handed quarks, then $\tilde{C}_{1}^{b d}$ dominates. For comparable left- and right-handed couplings all four Wilson coefficients, $C_{1,3}^{b d}, \tilde{C}_{1,3}^{b d}$, are important. The analogous discussion 

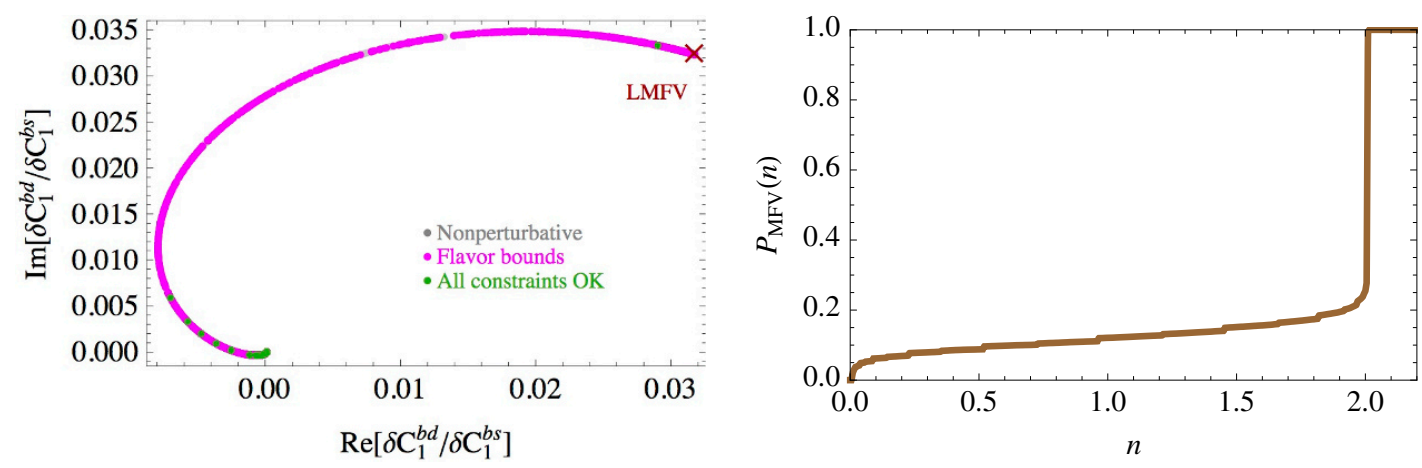

Figure 14. The FGB contributions to the $V-A$ current operator in the effective weak Hamiltonian. The left panel shows the values of the complex ratio $\delta C_{1}^{b d} / \delta C_{1}^{b s}$ for our scan points, with green points satisfying all constraints, magenta points excluded by flavor constraints and grey points by perturbativity considerations. The cross denotes the point $\delta C_{1}^{b d} / \delta C_{1}^{b s}=\left(V_{t d}^{*} / V_{t s}^{*}\right)^{2}$. In this point the MFV operator with the smallest number of Yukawa insertions completely dominates. The right panel shows the cumulative function $P_{\mathrm{MFV}}(n)$, see eq. (A.4).

applies to the case of FGB contributions to $B_{s}$ mixing obtained with a trivial $d \rightarrow s$ exchange.

As discussed in section 2 the contributions from the gauged flavor model is expandable in terms of the SM Yukawas. The contributions due to FGB exchanges can thus be written as

$$
\delta C_{1}^{b d}=c_{1}\left(y_{u} y_{u}^{\dagger}\right)_{13}^{2}+c_{2}\left(y_{u} y_{u}^{\dagger}\right)_{13}\left(y_{d} y_{d}^{\dagger} y_{u} y_{u}^{\dagger}\right)_{13}+\cdots=c_{1}\left(V_{t d}^{*} V_{t b}\right)^{2}+c_{2} y_{d}^{2}\left(V_{t d}^{*} V_{t b}\right)^{2}+\cdots,
$$

where $\left(y_{d}\right)_{i j}=\operatorname{diag}\left(y_{d}, y_{s}, y_{b}\right)$, and we set $y_{t}=1$ in the second equality. In eq. (A.3) we kept only the two terms relevant for the discussion below. The same expansion applies for $\delta C_{1}^{b s}$ with the replacement $d \rightarrow s$ in eq. (A.3).

In figure 14 (left) we show the ratio $\delta C_{1}^{b d} / \delta C_{1}^{b s}$, i.e. the NP contribution to the $V-A$ quark current operator due to tree-level FGB exchanges. Note that the ratio $\delta C_{1}^{b d} / \delta C_{1}^{b s}$ can be complex. If $c_{1}$, i.e. the leading MFV (LMFV) term, dominates then $\delta C_{1}^{b d} / \delta C_{1}^{b s} \simeq$ $\left(V_{t d}^{*} / V_{t s}^{*}\right)^{2}$. This is denoted by a cross in figure 14 (left). The addition of the operators with extra insertions of $y_{d} y_{d}^{\dagger}$ leads to $\delta C_{1}^{b d} / \delta C_{1}^{b s}$ not being equal to $\left(V_{t d}^{*} / V_{t s}^{*}\right)^{2}$. We verified that the curve for $\delta C_{1}^{b d} / \delta C_{1}^{b s}$ shown in figure 14 (left) can be fitted with the form of $\delta C_{1}^{b d, b s}$ in eq. (A.3) by taking $c_{1}$ real, $c_{2}$ complex, and varying $c_{1}$ from $\mathcal{O}(1)$ to vanishingly small. The points in our scan can be grouped into two sets. For the first set of points both $c_{1}$ and $c_{2}$ terms are sizeable. For the second set of points the $c_{1}$ term is negligible and $c_{2}$ dominates. This is shown in figure 14 (right), where we plot the cumulative distribution function

$$
P_{\mathrm{MFV}}(n)=\frac{N\left(\left|\delta C_{1}^{b d} / \delta C_{1}^{b s}\right| \geq\left(m_{d} / m_{s}\right)^{n}\left|V_{t d}^{*} / V_{t s}^{*}\right|^{2}\right)}{N_{\text {total }}} .
$$

The function $P_{\mathrm{MFV}}(n)$ can be interpreted as the fraction of points that have the ratio $\left|\delta C_{1}^{b d} / \delta C_{1}^{b s}\right|$ effectively dominated by operators with up to $y_{d}^{n}$ insertions. That is, the points dominated by the $c_{1}$ term contribute to $P_{\mathrm{MFV}}(0)$ (and to $P_{\mathrm{MFV}}(n)$ with $n \geq 0$ ), 

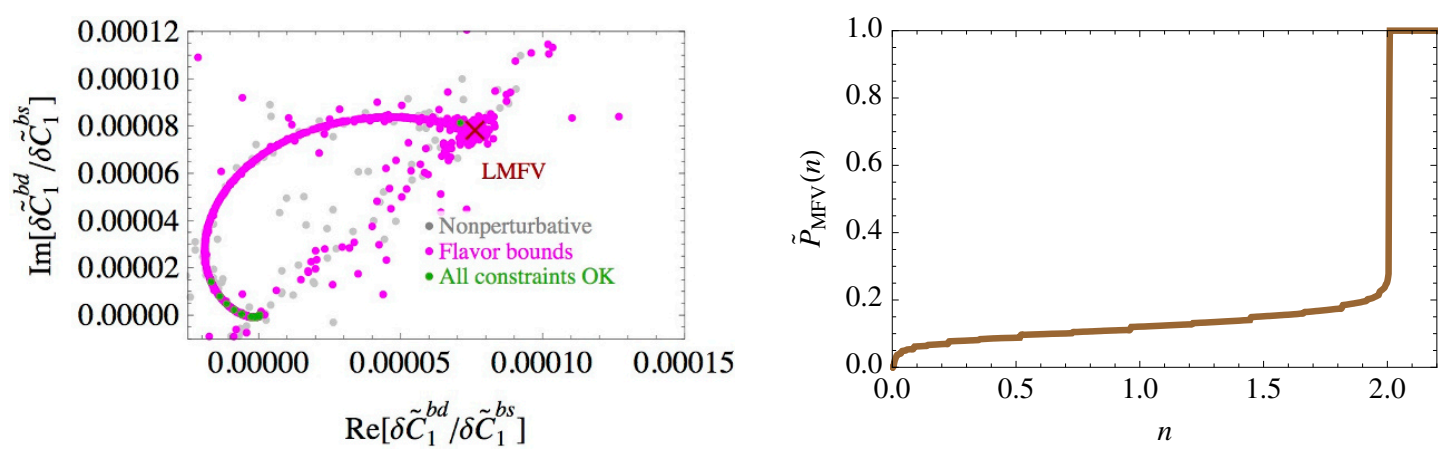

Figure 15. The FGB contributions to $V+A$ current operator in the effective weak Hamiltonian. Left panel shows the complex ratio $\delta \tilde{C}_{1}^{b d} / \delta \tilde{C}_{1}^{b s}$ for our scan points with the same color coding as in figure 14. The cross denotes the point $\delta \tilde{C}_{1}^{b d} / \delta \tilde{C}_{1}^{b s}=\left(m_{d} / m_{s}\right)^{2}\left(V_{t d}^{*} / V_{t s}^{*}\right)^{2}$, obtained if the MFV operator with the smallest number of Yukawa insertions dominates. The right panel shows the cumulative function $\tilde{P}_{\mathrm{MFV}}(n)$, see eq. (A.6).

while the points dominated by the $c_{2}$ term contribute to $P_{\mathrm{MFV}}(2)$ (and to $P_{\mathrm{MFV}}(n)$ with $n \geq 2$ ). The points with both $c_{1}$ and $c_{2}$ start contributing to $P_{\mathrm{MFV}}(n)$ for $n$ somewhere between 0 and 2, depending on the relative sizes of $c_{1}$ and $c_{2}$. Figure 14 (right) shows that the $c_{1}$ term dominates in a subleading (but nonzero) set of points, that about $10 \%$ points have both sizeable $c_{1}$ and $c_{2}$ terms, and that $c_{2}$ dominates in about $80 \%$ of the points.

The similar analysis can be performed for $V+A$ operators, $\tilde{Q}_{1}^{b d}$ and $\tilde{Q}_{1}^{b s}$. We expand the FGB contributions to their respective Wilson coefficients in terms of the SM Yukawas

$$
\begin{aligned}
\delta \tilde{C}_{1}^{b d} & =\tilde{c}_{1}\left(y_{d}^{\dagger} y_{u} y_{u}^{\dagger} y_{b}\right)_{13}^{2}+\tilde{c}_{2}\left(y_{d}^{\dagger} y_{u} y_{u}^{\dagger} y_{b}\right)_{13}\left(y_{d}^{\dagger} y_{d} y_{d}^{\dagger} y_{u} y_{u}^{\dagger} y_{b}\right)_{13}+\cdots \\
& =\tilde{c}_{1} y_{d}^{2} y_{b}^{2}\left(V_{t d}^{*} V_{t b}\right)^{2}+\tilde{c}_{2} y_{d}^{4} y_{b}^{2}\left(V_{t d}^{*} V_{t b}\right)^{2}+\cdots,
\end{aligned}
$$

and similarly for $\delta \tilde{C}_{1}^{b s}$ with the $d \rightarrow s$ replacement. We show in figure 15 (left) the ratio $\tilde{C}_{1}^{b d} / \tilde{C}_{1}^{b s}$ for our scan. If the $\tilde{c}_{1}$ term dominates, then $\tilde{C}_{1}^{b d} / \tilde{C}_{1}^{b s}=\left(m_{d} / m_{s}\right)^{2}\left(V_{t d}^{*} / V_{t s}^{*}\right)^{2}$, which is denoted by the cross in figure 15 (left). The points for which also the $\tilde{c}_{2}$ operator (and other operators denoted by ellipses above) is important then lie away from the $\tilde{C}_{1}^{b d} / \tilde{C}_{1}^{b s}=$ $\left(m_{d} / m_{s}\right)^{2}\left(V_{t d}^{*} / V_{t s}^{*}\right)^{2}$ region. We also define a cumulative function

$$
\tilde{P}_{\mathrm{MFV}}(n)=\frac{N\left(\left|\delta \tilde{C}_{1}^{b d} / \delta \tilde{C}_{1}^{b s}\right| \geq\left(m_{d} / m_{s}\right)^{n+2}\left|V_{t d}^{*} / V_{t s}^{*}\right|^{2}\right)}{N_{\text {total }}}
$$

The values for $\tilde{P}_{\mathrm{MFV}}(n)$ are shown in figure 15 (right). We see that also in this case the points cluster into two groups, with vanishing $\tilde{c}_{1}$ term or with both $\tilde{c}_{1}$ and $\tilde{c}_{2}$ relevant.

The above analysis demonstrates that the FGB contributions to the Wilson coefficients in the effective weak Hamiltonian can be expanded in terms of the SM Yukawas. This is a hallmark of (general) MFV. In particular, the expansion in terms of $m_{d, s} / m_{b}$ and offdiagonal CKM elements can still be performed and is not ruined by the large ratios of scales present in the problem such as the very disparate FGB masses. 


\section{B Thermal relic computation}

In this appendix we describe the calculation of relic density that was used in the scans in the main part of the paper. Several approximations to the coupled Boltzmann equations were necessary in order to reduce the evaluation time per benchmark and thus allow adequate coverage of the parameter space. We find the approximate solutions to be in agreement with the full solutions at the $\mathcal{O}(30 \%)$ level. The full numerical solution of the Boltzmann equations was obtained with MadDM [33] using a UFO model file [76], which was generated with the FeynRules package [34].

Denoting the DM multiplet by $\varphi$, where $\varphi$ is either a Dirac fermion or a complex scalar, the most general set of coupled Boltzmann equations reads [31]

$$
\begin{aligned}
\frac{d n_{\varphi_{i}}}{d t}+3 H n_{\varphi_{i}}= & -\sum_{j}\left\langle\sigma\left(\varphi_{i} \varphi_{j} \leftrightarrow X X\right) v_{\mathrm{lab}}\right\rangle\left(n_{\varphi_{i}} n_{\varphi_{j}}-n_{\varphi_{i}}^{\mathrm{EQ}} n_{\varphi_{j}}^{\mathrm{EQ}}\right) \\
& -\sum_{j \neq i}\left\langle\sigma\left(\varphi_{i} X \leftrightarrow \varphi_{j} X\right) v_{\mathrm{lab}}\right\rangle\left(n_{\varphi_{i}}-\frac{n_{\varphi_{i}}^{\mathrm{EQ}}}{n_{\varphi_{j}}^{\mathrm{EQ}}} n_{\varphi_{j}}\right) n_{X}^{\mathrm{EQ}} \\
& -\sum_{j \neq i}\left\langle\sigma\left(\varphi_{i} \varphi_{j} \leftrightarrow \varphi_{k} \varphi_{\ell}\right) v_{\mathrm{lab}}\right\rangle\left(n_{\varphi_{i}} n_{\varphi_{j}}-\frac{n_{\varphi_{i}}^{\mathrm{EQ}} n_{\varphi_{j}}^{\mathrm{EQ}}}{n_{\varphi_{k}}^{\mathrm{EQ}} \varphi_{\ell}^{\mathrm{EQ}}} n_{\varphi_{k}} n_{\varphi_{\ell}}\right) \\
& \pm \sum_{j \neq i}\left[\left\langle\Gamma\left(\varphi_{j, i} \rightarrow \varphi_{i, j} X\right)\right\rangle n_{\varphi_{j, i}}+\left\langle\sigma\left(\varphi_{j, i} X \rightarrow \varphi_{i, j}\right)\right\rangle n_{\varphi_{j, i}} n_{X}^{\mathrm{EQ}}\right],
\end{aligned}
$$

where $X$ denotes a generic SM state. For large mass splittings between the $\varphi$ components it is sufficient to consider the lightest $\varphi_{i}$ state in eq. (B.1). The contributions to the DM relic density from the heavy $\varphi$ components are exponentially suppressed by corresponding Boltzmann factors and can be neglected within our precision. In contrast, when the mass splittings are small the full set of coupled Boltzmann equations in eq. (B.1) needs to be considered. Nevertheless, even in this case several approximations are possible for our model, as we explain below.

First of all, the coannihilation of different $\varphi_{i}$ components into SM particles, $\varphi_{i} \varphi_{j} \rightarrow$ $X X(i \neq j)$, can be safely neglected in our model. In benchmarks that survive the experimental constraints the off-diagonal couplings of the lightest FGB to $\varphi$ are much smaller than the diagonal ones, see figure 16. Secondly, in the calculation of DM relic density we also neglect the flavor-changing DM scattering off the thermal background, $\varphi_{i} X \rightarrow \varphi_{j} X$. The $\varphi_{i} X \rightarrow \varphi_{j} X$ scattering can be important if $\left\langle\sigma\left(\varphi_{i} X \rightarrow \varphi_{j} X\right) v_{\mathrm{lab}}\right\rangle /\left\langle\sigma\left(\varphi_{i} \varphi_{j} \rightarrow X X\right) v_{\mathrm{lab}}\right\rangle \gtrsim$ $n_{\varphi_{j}} / n_{X}^{\mathrm{EQ}} \sim 10^{-9}$. In this case the off-diagonal couplings of $\mathcal{O}\left(10^{-4}\right)$ relative to the diagonal ones are in principle large enough to have $\mathcal{O}(1)$ effects on the relic density, and neglecting $\varphi_{i} X \rightarrow \varphi_{j} X$ may not be justified. Therefore, for the benchmarks with $\left(\hat{g}_{\chi}^{24}\right)_{23} /\left(\hat{g}_{\chi}^{24}\right)_{33}>3 \times 10^{-4}$ and small mass splittings among $\varphi$, we explicitly verified using MadDM that neglecting $\varphi_{i} X \rightarrow \varphi_{j} X$ scattering leads to a change in DM relic density smaller than $\mathcal{O}(30 \%)$.

Finally, we are able to neglect the pure DM scattering process in the third line of eq. (B.1) since $\left\langle\sigma\left(\varphi_{i} \varphi_{j} \leftrightarrow \varphi_{k} \varphi_{\ell}\right) v_{\text {lab }}\right\rangle \ll\left\langle\sigma\left(\varphi_{i} \varphi_{j} \leftrightarrow X X\right) v_{\text {lab }}\right\rangle$ in our model. The largest contribution to this process is from diagonal couplings between the FGB and 


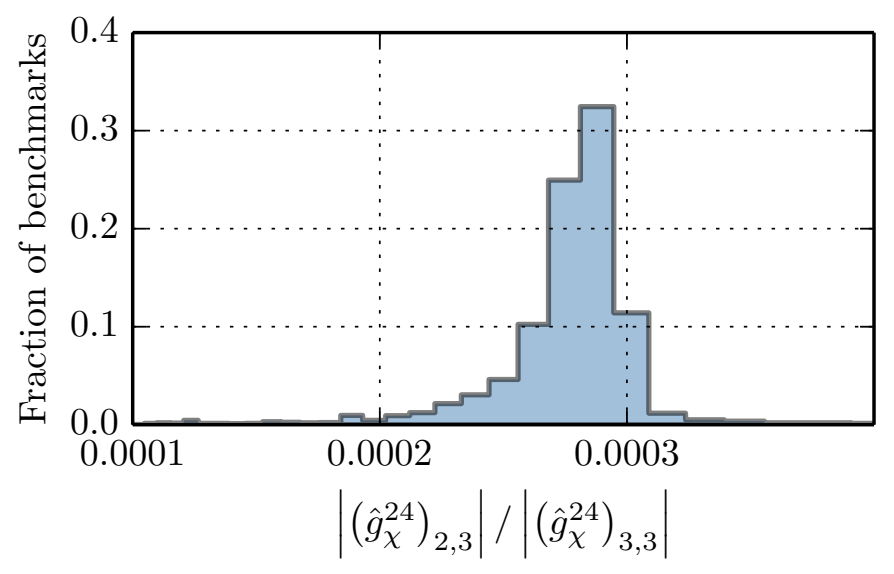

Figure 16. The fraction of benchmarks as a function of the off-diagonal couplings of the heaviest and next-to-heaviest DM components to the lightest FGB $\left(A^{24}\right)$ normalized by the diagonal coupling of the heaviest component.

DM. This process can couple the evolution of the DM species if $\left\langle\sigma\left(\varphi_{i} \varphi_{j} \leftrightarrow \varphi_{k} \varphi_{\ell}\right) v_{\text {lab }}\right\rangle \sim$ $\left\langle\sigma\left(\varphi_{i} \varphi_{j} \leftrightarrow X X\right) v_{\mathrm{lab}}\right\rangle$. The diagonal FGB couplings to the quarks and to the DM of the same generation are approximately equal. By accounting for the color factors and the multiplicity of channels when annihilating into SM fields one concludes that the pure DM scattering is indeed subleading.

Therefore, for almost mass degenerate $\varphi_{i}$ it is sufficient to consider a set of uncoupled Boltzmann equations

$$
\frac{d n_{\varphi_{i}}}{d t}+3 H n_{\varphi_{i}}=-\sum_{j}\left\langle\sigma\left(\varphi_{i} \varphi_{i} \leftrightarrow X X\right) v_{\mathrm{lab}}\right\rangle\left(n_{\varphi_{i}}^{2}-n_{\varphi_{i}}^{\mathrm{EQ} 2}\right) .
$$

The DM relic abundance is in this case the sum of relic abundances for each of the three components obtained from the above set of equations (the heavy $\varphi$ components, in our case $\varphi_{2}$ and $\varphi_{3}$, decay after their respective freeze-outs and contribute to the $\varphi_{1}$ DM relic abundance). In contrast, for large mass splittings the heavy $\varphi$ components are irrelevant for the calculation of the DM relic abundance. This is then obtained from eq. (B.2) by considering only the lightest DM state, in our case $\varphi_{1}$.

We calculate the DM relic abundance from eq. (B.2) using the freeze-out approximation [32], which gives

$$
\Omega h^{2}=\frac{1.07 \times 10^{9} \mathrm{GeV}^{-1}}{J\left(x_{f}\right) \sqrt{g_{*}} M_{P l}},
$$

where $M_{P l}=1.22 \times 10^{19} \mathrm{GeV}, g_{*}$ is the total number of effectively relativistic degrees of freedom at the time of the freeze-out, and

$$
J\left(x_{f}\right)=\int_{x_{f}}^{\infty} d x \frac{\left\langle\sigma v_{\mathrm{lab}}\right\rangle_{\mathrm{th}}}{x^{2}}
$$


The freeze-out temperature $\left(x_{f}=m_{\varphi_{1}} / T_{f}\right)$ is obtained by solving

$$
x_{f}=\ln \frac{0.038 g_{\mathrm{eff}} M_{P l} m_{\varphi_{1}}\left\langle\sigma v_{\mathrm{lab}}\right\rangle_{\mathrm{th}}}{\sqrt{g_{*} x_{f}}},
$$

where the thermally-averaged cross section is

$$
\left\langle\sigma v_{\mathrm{lab}}\right\rangle_{\mathrm{th}}=\frac{2 x^{\frac{3}{2}}}{\sqrt{\pi}} \int_{0}^{\infty} \sigma_{\mathrm{eff}} v_{\mathrm{lab}} \sqrt{\epsilon} e^{-x \epsilon} d \epsilon
$$

with $v_{\text {lab }}=2 \sqrt{\epsilon(1+\epsilon)} /(1+2 \epsilon)$ and $\epsilon=s /\left(2 m_{\varphi_{1}}\right)^{2}-1$. The freeze-out approximation is accurate to a few percent with respect to the full numerical solution of the Boltzmann equation [31].

The fermionic flavored DM annihilates through the s-channel exchange of FGBs. In this case, the integration over $x$ can be performed analytically and the double integral in eq. (B.4) reduces to a single one that can be efficiently evaluated numerically. In particular,

$$
J\left(x_{f}\right)=\int_{0}^{\infty} 2 \sigma v_{\mathrm{lab}} \operatorname{Erfc}\left(\sqrt{x_{f} \epsilon}\right) d \epsilon
$$

We evaluate the above integral numerically in the parameter scan.

In the annihilation cross section of the fermionic flavored DM we keep the dominant contribution - the $s$-channel exchange of the lightest FGB, $A^{24}$. The annihilation cross section for $\chi_{i} \bar{\chi}_{i} \rightarrow \bar{u}_{j} u_{j}$ (and similarly for $\chi_{i} \bar{\chi}_{i} \rightarrow \bar{d}_{j} d_{j}$ ) is given by

$$
\begin{aligned}
\sigma\left(\chi_{i} \bar{\chi}_{i} \rightarrow \bar{u}_{j} u_{j}\right)= & \frac{\left(\hat{g}_{\chi}^{24}\right)_{i i}^{2}}{4 \pi} \sqrt{\frac{s-4 m_{u_{j}}^{2}}{s-4 m_{\chi_{i}}^{2}}}\left(1+\frac{2 m_{\chi_{i}}^{2}}{s}\right) \times \\
& \times \frac{\left(\hat{\mathcal{G}}_{V}^{u}\right)_{j j, 24}^{2}\left(s+2 m_{u_{j}}^{2}\right)+\left(\hat{\mathcal{G}}_{A}^{u}\right)_{j j, 24}^{2}\left(s-4 m_{u_{j}}^{2}\right)}{\left(s-m_{A^{24}}^{2}\right)^{2}+m_{A^{24}}^{2} \Gamma_{A^{24}}^{2}}
\end{aligned}
$$

where the vector and axial-vector couplings to quarks, $\hat{\mathcal{G}}_{A, V}$, were defined in eq. $(4.2), \sqrt{s}$ is the center-of-mass energy and $\Gamma_{A^{24}}$ is the total decay width of the lightest FGB. The decay rate for $A^{24} \rightarrow \bar{u}_{j} u_{j}$ assuming $m_{A^{24}}>2 m_{u_{j}}$ is

$$
\Gamma\left(A^{24} \rightarrow \bar{u}_{j} u_{j}\right)=\frac{m_{A}^{24}}{4 \pi} \sqrt{1-\frac{4 m_{u_{j}}^{2}}{m_{A^{24}}^{2}}}\left[\left(\hat{\mathcal{G}}_{V}^{u}\right)_{j j, 24}^{2}\left(1+\frac{2 m_{u_{j}}^{2}}{m_{A^{24}}^{2}}\right)+\left(\hat{\mathcal{G}}_{A}^{u}\right)_{j j, 24}^{2}\left(1-\frac{4 m_{u_{j}}^{2}}{m_{A^{24}}^{2}}\right)\right] .
$$

The rate for $A^{24} \rightarrow \chi_{i} \bar{\chi}_{i}, \bar{d}_{j} d_{j}$ is obtained after trivial replacements for masses and couplings (and dividing by the $N_{c}$ color factor for decays to $\chi_{i} \bar{\chi}_{i}$ ). The total FGB decay rate is obtained after summing over all kinematically allowed decay channels. 


\section{Higgs coupling Feynman rules}

As noted in section 4.5, the $h \bar{f} f$ Feynman rules given in appendix A.1 of ref. [30] contain a typo. The corrected Feynman rules are given here.

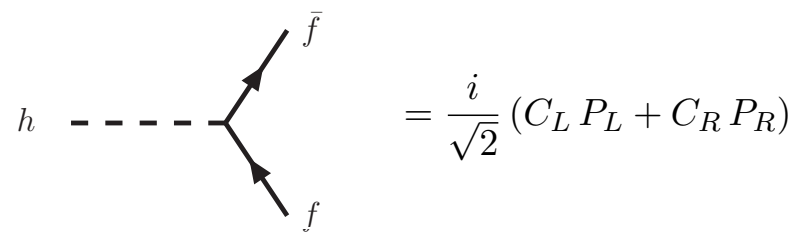

where the couplings $C_{L}$ and $C_{R}$ are:

$$
\begin{aligned}
h \bar{u}_{i} u_{i} & : C_{L}=C_{R}=+\lambda_{u} s_{u_{R} i} c_{u_{L} i} \\
h \bar{u}_{i}^{\prime} u_{i}^{\prime} & : C_{L}=C_{R}=-\lambda_{u} c_{u_{R} i} s_{u_{L} i} \\
h \bar{u}_{i} u_{i}^{\prime} & :\left\{\begin{array}{l}
C_{R}=-\lambda_{u} c_{u_{R} i} c_{u_{L} i} \\
C_{L}=+\lambda_{u} s_{u_{R}} s_{u_{L} i}
\end{array}\right. \\
h \bar{u}_{i}^{\prime} u_{i} & :\left\{\begin{array}{l}
C_{R}=+\lambda_{u} s_{u_{R} i} s_{u_{L} i} \\
C_{L}=-\lambda_{u} c_{u_{R} i} c_{u_{L} i}
\end{array}\right.
\end{aligned}
$$

Open Access. This article is distributed under the terms of the Creative Commons Attribution License (CC-BY 4.0), which permits any use, distribution and reproduction in any medium, provided the original author(s) and source are credited.

\section{References}

[1] G. Jungman, M. Kamionkowski and K. Griest, Supersymmetric dark matter, Phys. Rept. 267 (1996) 195 [hep-ph/9506380] [INSPIRE].

[2] M. Hirsch, S. Morisi, E. Peinado and J.W.F. Valle, Discrete dark matter, Phys. Rev. D 82 (2010) 116003 [arXiv: 1007.0871] [INSPIRE].

[3] M.S. Boucenna, M. Hirsch, S. Morisi, E. Peinado, M. Taoso and J.W.F. Valle, Phenomenology of Dark Matter from $A_{4}$ Flavor Symmetry, JHEP 05 (2011) 037 [arXiv: 1101.2874] [INSPIRE].

[4] M.S. Boucenna, S. Morisi, E. Peinado, Y. Shimizu and J.W.F. Valle, Predictive discrete dark matter model and neutrino oscillations, Phys. Rev. D 86 (2012) 073008 [arXiv:1204.4733] [INSPIRE].

[5] D. Meloni, S. Morisi and E. Peinado, Neutrino phenomenology and stable dark matter with A4, Phys. Lett. B 697 (2011) 339 [arXiv:1011.1371] [InSPIRE].

[6] M. Lindner, D. Schmidt and T. Schwetz, Dark Matter and neutrino masses from global $U(1)_{B-L}$ symmetry breaking, Phys. Lett. B 705 (2011) 324 [arXiv:1105.4626] [INSPIRE].

[7] B. Batell, J. Pradler and M. Spannowsky, Dark Matter from Minimal Flavor Violation, JHEP 08 (2011) 038 [arXiv:1105.1781] [INSPIRE].

[8] B. Batell, T. Lin and L.-T. Wang, Flavored Dark Matter and R-Parity Violation, JHEP 01 (2014) 075 [arXiv:1309.4462] [INSPIRE]. 
[9] L. Lopez-Honorez and L. Merlo, Dark matter within the minimal flavour violation ansatz, Phys. Lett. B 722 (2013) 135 [arXiv: 1303.1087] [INSPIRE].

[10] B. Grinstein, M. Redi and G. Villadoro, Low Scale Flavor Gauge Symmetries, JHEP 11 (2010) 067 [arXiv: 1009.2049] [INSPIRE].

[11] P. Agrawal, B. Batell, D. Hooper and T. Lin, Flavored Dark Matter and the Galactic Center Gamma-Ray Excess, Phys. Rev. D 90 (2014) 063512 [arXiv:1404.1373] [InSPIRE].

[12] C.-J. Lee and J. Tandean, Lepton-Flavored Scalar Dark Matter with Minimal Flavor Violation, JHEP 04 (2015) 174 [arXiv:1410.6803] [INSPIRE].

[13] F. Bishara and J. Zupan, Continuous Flavor Symmetries and the Stability of Asymmetric Dark Matter, JHEP 01 (2015) 089 [arXiv: 1408.3852] [INSPIRE].

[14] P. Agrawal, M. Blanke and K. Gemmler, Flavored dark matter beyond Minimal Flavor Violation, JHEP 10 (2014) 72 [arXiv:1405.6709] [INSPIRE].

[15] P. Agrawal, S. Blanchet, Z. Chacko and C. Kilic, Flavored Dark Matter and Its Implications for Direct Detection and Colliders, Phys. Rev. D 86 (2012) 055002 [arXiv:1109.3516] [INSPIRE].

[16] A. Hamze, C. Kilic, J. Koeller, C. Trendafilova and J.-H. Yu, Lepton-Flavored Asymmetric Dark Matter and Interference in Direct Detection, Phys. Rev. D 91 (2015) 035009 [arXiv: 1410.3030] [INSPIRE].

[17] A. Kumar and S. Tulin, Top-flavored dark matter and the forward-backward asymmetry, Phys. Rev. D 87 (2013) 095006 [arXiv: 1303.0332] [INSPIRE].

[18] J.F. Kamenik and J. Zupan, Discovering Dark Matter Through Flavor Violation at the LHC, Phys. Rev. D 84 (2011) 111502 [arXiv:1107.0623] [INSPIRE].

[19] J. Kile, A. Kobach and A. Soni, Lepton-Flavored Dark Matter, Phys. Lett. B 744 (2015) 330 [arXiv: 1411.1407] [INSPIRE].

[20] J. Kile and A. Soni, Flavored Dark Matter in Direct Detection Experiments and at LHC, Phys. Rev. D 84 (2011) 035016 [arXiv:1104.5239] [InSPIRE].

[21] L. Calibbi, A. Crivellin and B. Zaldivar, The Flavour Portal to Dark Matter, arXiv: 1501.07268 [INSPIRE].

[22] G. D'Ambrosio, G.F. Giudice, G. Isidori and A. Strumia, Minimal flavor violation: An Effective field theory approach, Nucl. Phys. B 645 (2002) 155 [hep-ph/0207036] [INSPIRE].

[23] R.S. Chivukula and H. Georgi, Composite Technicolor Standard Model, Phys. Lett. B 188 (1987) 99 [INSPIRE].

[24] E. Gabrielli and G.F. Giudice, Supersymmetric corrections to epsilon prime/epsilon at the leading order in QCD and QED, Nucl. Phys. B 433 (1995) 3 [Erratum ibid. B 507 (1997) 549] [hep-lat/9407029] [INSPIRE].

[25] A. Ali and D. London, Profiles of the unitarity triangle and CP-violating phases in the standard model and supersymmetric theories, Eur. Phys. J. C 9 (1999) 687 [hep-ph/9903535] [INSPIRE].

[26] A.J. Buras, P. Gambino, M. Gorbahn, S. Jager and L. Silvestrini, Universal unitarity triangle and physics beyond the standard model, Phys. Lett. B 500 (2001) 161 [hep-ph/0007085] [INSPIRE]. 
[27] A.J. Buras, Minimal flavor violation, Acta Phys. Polon. B 34 (2003) 5615 [hep-ph/0310208] [INSPIRE].

[28] A.L. Kagan, G. Perez, T. Volansky and J. Zupan, General Minimal Flavor Violation, Phys. Rev. D 80 (2009) 076002 [arXiv:0903.1794] [INSPIRE].

[29] C. Smith, Proton stability from a fourth family, Phys. Rev. D 85 (2012) 036005 [arXiv: 1105.1723] [INSPIRE].

[30] A.J. Buras, M.V. Carlucci, L. Merlo and E. Stamou, Phenomenology of a Gauged SU $(3)^{3}$ Flavour Model, JHEP 03 (2012) 088 [arXiv:1112.4477] [INSPIRE].

[31] P. Gondolo and G. Gelmini, Cosmic abundances of stable particles: Improved analysis, Nucl. Phys. B 360 (1991) 145 [inSPIRE].

[32] K. Griest and D. Seckel, Three exceptions in the calculation of relic abundances, Phys. Rev. D 43 (1991) 3191 [INSPIRE].

[33] M. Backovic, K. Kong and M. McCaskey, MadDM v.1.0: Computation of Dark Matter Relic Abundance Using MadGraph5, Physics of the Dark Universe 5-6 (2014) 18 [arXiv: 1308.4955] [INSPIRE].

[34] A. Alloul, N.D. Christensen, C. Degrande, C. Duhr and B. Fuks, FeynRules 2.0 - A complete toolbox for tree-level phenomenology, Comput. Phys. Commun. 185 (2014) 2250 [arXiv: 1310.1921] [INSPIRE].

[35] B. Fields and S. Sarkar, Big-Bang nucleosynthesis (2006 Particle Data Group mini-review), astro-ph/0601514 [INSPIRE].

[36] W. Hu and J. Silk, Thermalization and spectral distortions of the cosmic background radiation, Phys. Rev. D 48 (1993) 485 [INSPIRE].

[37] W. Hu and J. Silk, Thermalization constraints and spectral distortions for massive unstable relic particles, Phys. Rev. Lett. 70 (1993) 2661 [INSPIRE].

[38] R. Essig, E. Kuflik, S.D. McDermott, T. Volansky and K.M. Zurek, Constraining Light Dark Matter with Diffuse X-Ray and Gamma-Ray Observations, JHEP 11 (2013) 193 [arXiv: 1309.4091] [INSPIRE].

[39] F. Iocco, G. Mangano, G. Miele, O. Pisanti and P.D. Serpico, Primordial Nucleosynthesis: from precision cosmology to fundamental physics, Phys. Rept. 472 (2009) 1 [arXiv:0809.0631] [INSPIRE].

[40] D. Lindley, Cosmological Constraints on the Lifetime of Massive Particles, Astrophys. J. 294 (1985) 1 [INSPIRE].

[41] M.H. Reno and D. Seckel, Primordial Nucleosynthesis: The Effects of Injecting Hadrons, Phys. Rev. D 37 (1988) 3441 [INSPIRE].

[42] S. Dimopoulos, R. Esmailzadeh, L.J. Hall and G.D. Starkman, Is the Universe Closed by Baryons? Nucleosynthesis With a Late Decaying Massive Particle, Astrophys. J. 330 (1988) 545 [INSPIRE].

[43] R.J. Scherrer and M.S. Turner, Primordial Nucleosynthesis with Decaying Particles. 1. Entropy Producing Decays. 2. Inert Decays, Astrophys. J. 331 (1988) 19 [INSPIRE].

[44] J.R. Ellis, G.B. Gelmini, J.L. Lopez, D.V. Nanopoulos and S. Sarkar, Astrophysical constraints on massive unstable neutral relic particles, Nucl. Phys. B 373 (1992) 399 [INSPIRE]. 
[45] M. Kawasaki, K. Kohri and T. Moroi, Big-Bang nucleosynthesis and hadronic decay of long-lived massive particles, Phys. Rev. D 71 (2005) 083502 [astro-ph/0408426] [INSPIRE].

[46] G. Bélanger, F. Boudjema, A. Pukhov and A. Semenov, Dark matter direct detection rate in a generic model with MicrOMEGAs 2.2, Comput. Phys. Commun. 180 (2009) 747 [arXiv:0803.2360] [INSPIRE].

[47] G. Arcadi, Y. Mambrini, M.H.G. Tytgat and B. Zaldivar, Invisible $Z^{\prime}$ and dark matter: LHC vs LUX constraints, JHEP 03 (2014) 134 [arXiv:1401.0221] [INSPIRE].

[48] LUX collaboration, D.S. Akerib et al., First results from the LUX dark matter experiment at the Sanford Underground Research Facility, Phys. Rev. Lett. 112 (2014) 091303 [arXiv: 1310.8214] [INSPIRE].

[49] A. Urbano and W. Xue, Constraining the Higgs portal with antiprotons, JHEP 03 (2015) 133 [arXiv: 1412.3798] [INSPIRE].

[50] J.M. Cline and K. Kainulainen, Electroweak baryogenesis and dark matter from a singlet Higgs, JCAP 01 (2013) 012 [arXiv:1210.4196] [INSPIRE].

[51] P. Junnarkar and A. Walker-Loud, Scalar strange content of the nucleon from lattice QCD, Phys. Rev. D 87 (2013) 114510 [arXiv:1301.1114] [INSPIRE].

[52] J.M. Alarcon, J. Martin Camalich and J.A. Oller, The chiral representation of the $\pi N$ scattering amplitude and the pion-nucleon sigma term, Phys. Rev. D 85 (2012) 051503 [arXiv:1110.3797] [INSPIRE].

[53] L. Alvarez-Ruso, T. Ledwig, J. Martin Camalich and M. Vicente Vacas, Nucleon mass and pion-nucleon sigma term from a chiral analysis of lattice QCD world data, EPJ Web Conf. 73 (2014) 04015.

[54] A. Crivellin, F. D'Eramo and M. Procura, New Constraints on Dark Matter Effective Theories from Standard Model Loops, Phys. Rev. Lett. 112 (2014) 191304 [arXiv: 1402.1173] [INSPIRE].

[55] A. Crivellin, M. Hoferichter and M. Procura, Accurate evaluation of hadronic uncertainties in spin-independent WIMP-nucleon scattering: Disentangling two- and three-flavor effects, Phys. Rev. D 89 (2014) 054021 [arXiv:1312.4951] [INSPIRE].

[56] M. Boudaud, M. Cirelli, G. Giesen and P. Salati, A fussy revisitation of antiprotons as a tool for Dark Matter searches, JCAP 05 (2015) 013 [arXiv: 1412.5696] [INSPIRE].

[57] O. Adriani et al., Measurement of the flux of primary cosmic ray antiprotons with energies of 60-MeV to 350-GeV in the PAMELA experiment, JETP Lett. 96 (2013) 621 [INSPIRE].

[58] Fermi-LAT collaboration, M. Ackermann et al., Searching for Dark Matter Annihilation from Milky Way Dwarf Spheroidal Galaxies with Six Years of Fermi-LAT Data, arXiv: 1503.02641 [INSPIRE].

[59] M.R. Buckley et al., Search for Gamma-ray Emission from Dark Matter Annihilation in the Large Magellanic Cloud with the Fermi Large Area Telescope, Phys. Rev. D 91 (2015) 102001 [arXiv: 1502.01020] [INSPIRE].

[60] Fermi LAT collaboration, M. Ackermann et al., Limits on Dark Matter Annihilation Signals from the Fermi LAT 4-year Measurement of the Isotropic Gamma-Ray Background, arXiv: 1501.05464 [INSPIRE]. 
[61] ATLAS collaboration, Search for new phenomena in the dijet mass distribution using $p-p$ collision data at $\sqrt{s}=8 \mathrm{TeV}$ with the ATLAS detector, Phys. Rev. D 91 (2015) 052007 [arXiv: 1407.1376] [INSPIRE].

[62] CMS collaboration, Inclusive search for a vector-like T quark with charge $\frac{2}{3}$ in pp collisions at $\sqrt{s}=8 \mathrm{TeV}$, Phys. Lett. B 729 (2014) 149 [arXiv:1311.7667] [INSPIRE].

[63] E. Eichten, I. Hinchliffe, K.D. Lane and C. Quigg, Super Collider Physics, Rev. Mod. Phys. 56 (1984) 579 [INSPIRE].

[64] K.D. Lane and M.V. Ramana, Walking technicolor signatures at hadron colliders, Phys. Rev. D 44 (1991) 2678 [INSPIRE].

[65] A.J. Buras, S. Jager and J. Urban, Master formulae for Delta F=2 NLO QCD factors in the standard model and beyond, Nucl. Phys. B 605 (2001) 600 [hep-ph/0102316] [INSPIRE].

[66] J. Laiho, E. Lunghi and R.S. Van de Water, Lattice QCD inputs to the CKM unitarity triangle analysis, Phys. Rev. D 81 (2010) 034503 [arXiv:0910.2928] [InSPIRE].

[67] A.J. Buras, D. Guadagnoli and G. Isidori, On $\epsilon_{K}$ Beyond Lowest Order in the Operator Product Expansion, Phys. Lett. B 688 (2010) 309 [arXiv:1002.3612] [INSPIRE].

[68] A.J. Buras and D. Guadagnoli, Correlations among new CP-violating effects in $\Delta F=2$ observables, Phys. Rev. D 78 (2008) 033005 [arXiv:0805.3887] [INSPIRE].

[69] J. Brod and M. Gorbahn, Next-to-Next-to-Leading-Order Charm-Quark Contribution to the CP-violation Parameter $\epsilon_{K}$ and $\Delta M_{K}$, Phys. Rev. Lett. 108 (2012) 121801 [arXiv:1108.2036] [INSPIRE].

[70] J. Brod and M. Gorbahn, $\epsilon_{K}$ at Next-to-Next-to-Leading Order: The Charm-Top-Quark Contribution, Phys. Rev. D 82 (2010) 094026 [arXiv: 1007.0684] [INSPIRE].

[71] A.J. Buras, L. Merlo and E. Stamou, The Impact of Flavour Changing Neutral Gauge Bosons on $\bar{B} \rightarrow X_{s} \gamma$, JHEP 08 (2011) 124 [arXiv: 1105.5146] [INSPIRE].

[72] M. Misiak et al., Estimate of $\mathcal{B}\left(\bar{B} \rightarrow X_{s} \gamma\right)$ at $\mathcal{O}\left(\alpha_{s}^{2}\right)$, Phys. Rev. Lett. 98 (2007) 022002 [hep-ph/0609232] [INSPIRE].

[73] P. Gambino and M. Misiak, Quark mass effects in $\bar{B} \rightarrow X_{s} \gamma$, Nucl. Phys. B 611 (2001) 338 [hep-ph/0104034] [INSPIRE].

[74] M. Misiak and M. Steinhauser, NNLO QCD corrections to the $\bar{B} \rightarrow X_{s} \gamma$ matrix elements using interpolation in $m_{c}$, Nucl. Phys. B 764 (2007) 62 [hep-ph/0609241] [INSPIRE].

[75] UTFIT collaboration, M. Bona et al., Model-independent constraints on $\Delta F=2$ operators and the scale of new physics, JHEP 03 (2008) 049 [arXiv:0707.0636] [INSPIRE].

[76] C. Degrande, C. Duhr, B. Fuks, D. Grellscheid, O. Mattelaer and T. Reiter, UFO - The Universal FeynRules Output, Comput. Phys. Commun. 183 (2012) 1201 [arXiv:1108. 2040] [INSPIRE]. 